\title{
A novel discrete element method based on the distance potential for arbitrary 2D convex elements
}

\author{
Lanhao Zhao ${ }^{\mathrm{a}}$, Xunnan $\mathrm{Liu}^{\mathrm{a},{ }^{*}}$, Jia Mao ${ }^{\mathrm{a}}$, Dong Xu ${ }^{\mathrm{b}}$, Antonio Munjiza ${ }^{\mathrm{c}}$, Eldad Avital ${ }^{\mathrm{d}}$ \\ ${ }^{a}$ College of Water Conservancy and Hydropower, Hohai University, Nanjing, 210098, China

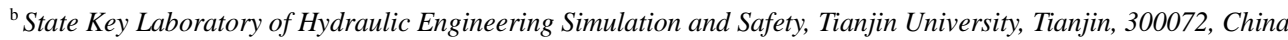 \\ ${ }^{\mathrm{c}}$ Faculty of Civil Engineering, University of Split, Croatia \\ ${ }^{\mathrm{d}}$ School of Engineering and Materials Science, Queen Mary University of London, London, E1 4NS, United Kingdom
}

\begin{abstract}
SUMMARY
A new two-dimensional discrete element method, which is able to simulate a system involving a large number of arbitrary convex elements, is proposed. In this approach, a novel distance potential function is defined using a normalized format of the penetrated distance between contact couples, whilst a holonomic and precise algorithm for contact interaction is established, accounting for the influence of the tangential contact force. Furthermore, the new contact detection algorithm is well suited for nonuniform blocks unlike the common No Binary Search (NBS) method that requires uniform elements. The proposed method retains the merit of the combined finite-discrete element method (FDEM) and avoids its deficiencies. Compared with the existing FDEM, the distance potential function has a clear physical meaning, where the calculation of contact interaction avoids the influence of the element shape. Accordingly, the new method completely gets rid of the restraint of uniform element type and can be applied to arbitrary convex elements. The new method is validated with well-known benchmark examples and the results are in very good agreement with existing experimental measurement and analytical solutions. Finally, the proposed method is applied to simulate the Tangjiashan landslide.
\end{abstract}

KEY WORDS: discrete element method; time integration, explicit; contact; arbitrary convex polygonal blocks; distance potential function

* Corresponding author:

Xunnan LIU

Tel.: +86 15195856602

Fax: +86 2583786281

E-mail address: liuxunnan@hhu.edu.cn 


\section{Introduction}

Discontinuous problems exist in many practical engineering scenarios, for instance, underground mining, rock blasting, tunneling. The problem involves a large number of individual particles or blocks in the system and it is challenging to determine the contact interaction between the particles and blocks when using the continuum assumption as in the finite element method (FEM) and the boundary element method (BEM).

Over recent decades, the development of accurate and efficient numerical methods for discontinuous problems has become a very active research field. Depending upon the formulation and solution for the dynamic equation, these methods can be grouped into the implicit and explicit approaches The most representative method of the implicit approach is the discontinuous deformation analysis (DDA). Since the initiation of the DDA by Shi [1] in 1985, it has achieved various developments [2-4]. Many studies have been carried out to validate the performance of this method [5-8]. However, it still suffers from various deficiencies. The contact condition is determined by the process of an open-close iteration which repeatedly fixes and removes normal and tangential springs between the blocks. As a result, the stiffness of contact springs affects the results significantly [9]. Another drawback is that the numerical accuracy is sensitive to the choice of the penalty value and the time step size [10]. Furthermore, DDA is not suitable for irregular blocks [11] and it has no efficient way to solve the corner-to-corner contact condition [4]. Originally, DDA cannot reflect the accurate stress distribution within the blocks. To address this problem, Shi [12] proposed the numerical manifold method (NMM) which is well suited for the continuous-discontinuous problem [13-16]. However, the NMM is still in its infancy and suffers from some inherent deficiencies, such as, the linear dependence problem [17] and is insufficiently effective for contact defection and contact transfer processes.

An alternative to the above discontinuous numerical methods is the discrete element method (DEM) Rather than the implicit approach utilized in DDA and NMM, DEM relies on an explicit approach to avoid solving a matrix of dynamic motion equations. Besides, the non-linearity property in the material behavior can also be handled in a straightforward manner [11]. Generally, DEM is divided into the granular discrete method and the block discrete element method according to the geometrical characteristic of the element. The granular discrete element method, firstly applied to analyze the movement of soils, has enjoyed considerable popularity due to the simplicity of the element type [18] and efficiency in contact detection [19], since it was suggested by Cundall and Strack [20]. Over the past twenty years, the method has been widely applied to many different fields such as the mechanism of granular materials [21-23], and rock fracture [24-26]. However, it has a severe drawback that the physical behavior of a single particle model as well as the large assemblies can be different from the actual behavior in some applied situations [27]. For example, interlocking of the particle cannot be captured with circular or spherical elements $[28,29]$. In addition, the value of the shear strength among the 
granular particle assemblies are below those obtained experimentally [30].

On the other hand, the block discrete element method has received a close attention in recent years because it considers the realistic element shape. This kind of method was firstly raised by Cundall and Strack [31] in 1971 for the analysis of progressive failure of rock slopes. The core concept of this method is that it treats all the blocks as rigid and a small amount of penetration is allowed in order to consider the relative displacement. At each time step, the contact interaction is updated by the incremental forcedisplacement law as shown in Figure 1. The application of this method is limited initially due to the various categories of contact situations, such as, vertex-to-vertex, vertex-to-edge and edge-to-edge contacts. In order to solve the contact problem, Cundall introduced the common plane (CP) method [32] which is now widely applied in various types of discrete element method codes. By translating and rotating the common plane, the contact types can be reduced to corner-to-plane contact. In recent years, the discrete element method with a polygon has been developed in the UDEC [33], and has been successfully employed in many applications [34-37].
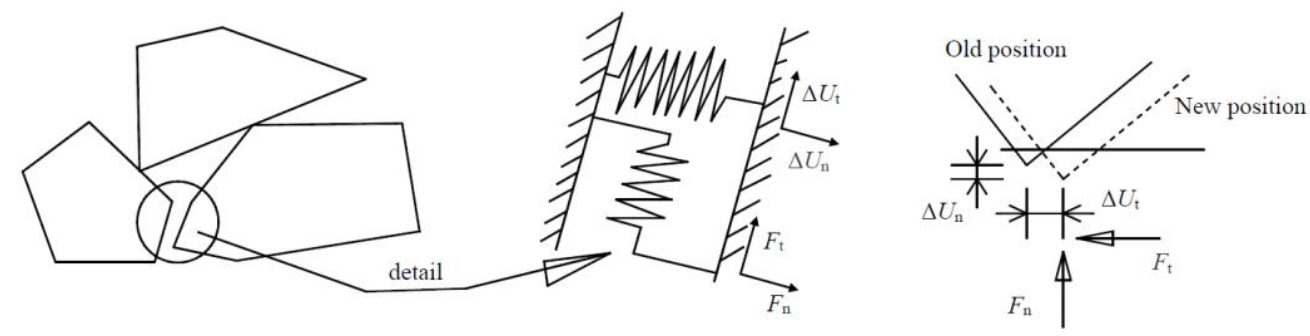

Figure 1. Mechanical model of the contact force in 2D DEM [11].

However this method has an incomplete contact interaction algorithm because of a lack of simple and unified mechanical model for different contact situations. In addition, it cannot deal with the vertexto-vertex contact. For the standard block DEM method, this kind of difficulty mainly comes from the non-determinacy of the normal contact force, as the normal direction is evolved discontinuously and not smoothly at the corner. This also results in an inconsistent contact force, which could cause energy imbalance and numerical errors [38]. One common practice to overcome the corner singularity is the application of a corner rounding procedure so that the blocks can slide past one another in a smooth way when two opposing corners interact [4, 39]. Even though the common plane method and the improved algorithm have been proposed to solve the contact detection problem, they still face a number of problems, such as the proper choice of the common plane, the iterative error for the spinning common plane and the uniqueness of the plane. Consequently, the reliability of simulation results is influenced by these shortcomings of standard DEM.

In recent years, some improvements have been made to make DEM more accordant with practical circumstances and provide more efficient models of contact interaction [18, 39-44] and contact detection algorithm [45-51]. Among them, the combined finite-discrete element method (FDEM) draws the most 
attention since it was introduced by Munjiza [52]. In FDEM, the medium is discretized into a large number of triangular elements with a similar size. The contact interaction is determined by overlapping areas between the contact couples with a new definition of potential function. According to the Green's formula and properties of a linear potential distribution in the triangular element, the calculation of the normal contact force is simplified as an integral of the potential on the boundary of the overlapping area. Moreover, the direction of the normal contact force can also be defined based on the outer normal direction of the boundary. In general, Munjiza has made a revolutionary impact on the model of contact interaction which introduces a new and uniform calculation model to avoid tackling different contact situations as the vertex-to-vertex contact and energy conservation is also obtained during the contact process. On the other hand, Munjiza [53] introduced the no binary search (NBS) method which reduces the CPU and computer memory requirements for processing contact problems with a large number of separate elements[38]. In recent years, a two-dimensional research code Y-code has been developed [38], together with a graphical user interface (Y-GUI) [54]. It has been successfully employed in various applications [55-60].

Nevertheless, there are some aspects that need to be strengthened in this method. The definition of the potential function lacks a clear physical meaning. In addition, both the value of the potential function and its normal contact force are greatly influenced by the element shape. In other words, with the same penetration and overlapping areas, the potential value and the normal contact force are not the same at all time. This method also does not include the calculation of the tangential contact force due to the difficult determination of the tangential direction at each time step. Another drawback is this method cannot be utilized for an arbitrary polygonal element. Moreover, the efficiency of the NBS algorithm is significantly influenced, when it is applied for a problem that includes a large number of elements with different sizes.

In the current work, a new two-dimensional discrete element method is proposed. The basic idea of the proposed method is motivated by the limited application of FDEM due to its sensitiveness to the element shape and omission of the tangential contact force. In this approach, a novel definition of a distance potential function is developed and a complete calculation algorithm for contact interaction is exhibited. Furthermore, a non-uniform block contact detection algorithm is introduced to overcome the defects of the NBS contact detection algorithm. In comparison with FDEM, the new approach exhibits a clearer physical meaning for the distance potential function and presents a more accurate calculation of contact interaction for arbitrary convex polygonal elements. In addition, the cost of the developed contact detection algorithm is proportional to the total number of individual blocks. As a result, it is possible for the proposed method to be utilized in large-scale discrete element simulations.

This paper is organized as follows. In the next section, after the basic idea of FDEM is given, the concept of a potential function is introduced. Then the distance potential function and the algorithm of 
contact interaction are established in detail in section 3. The non-uniform block discrete element contact detection algorithm is introduced in section 4. Several illustrative examples are studied to validate the presented method in section 5. Conclusions are summarized in section 6.

\section{Basic idea of potential contact interaction}

In FDEM, the system is represented by individual blocks and each block is discretized into finite elements. With the definition of the potential function, FDEM provides an effective way to simulate the process of transition from continuum to discontinuum. In this section, the basic definition of a potential function and the calculation algorithm of the potential contact interaction are introduced in detail.

\subsection{Potential contact force}

As described in FDEM, contact couples penetrate each other and this results in a distributed contact force which is generally associated with the shape and size of the overlapping area between the contact couples.

Consider two discrete elements, $\beta_{t}$ and $\beta_{c}$, with arbitrary shapes. $\beta_{t}$ is penetrated by $\beta_{c}$ as shown in Figure 2. The overlapping area $S$ is bounded by the boundary $\Gamma$.

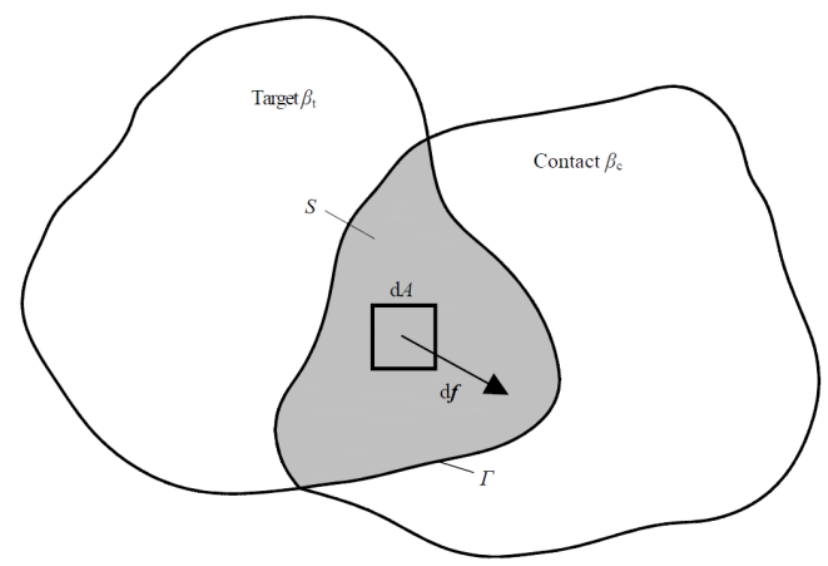

Figure 2. Two contact elements and the contact force $\mathrm{d} f$ due to the infinitesimal overlapping area $\mathrm{d} A$.

Considering an infinitesimal overlapping area $\mathrm{d} A$, the infinitesimal normal contact force is obtained:

$$
\mathrm{d} f=\left[\operatorname{grad} \varphi_{c}-\operatorname{grad} \varphi_{t}\right],
$$

where $\varphi_{c}$ and $\varphi_{t}$ are the values of a potential function at a point in $\mathrm{d} A$ belonging to $\beta_{c}$ and $\beta_{t}$, respectively.

Thus, the total normal contact force over the overlapping area is generated:

$$
\boldsymbol{f}_{n}=\int_{S}\left[\operatorname{grad} \varphi_{c}-\operatorname{grad} \varphi_{t}\right] \mathrm{d} A
$$

This can also be rewritten as an integral over the boundary $\Gamma$ in accordance with Green Formula: 


$$
\boldsymbol{f}_{n}=\int_{\Gamma} \boldsymbol{n}_{\Gamma}\left(\varphi_{c}-\varphi_{t}\right) \mathrm{d} \Gamma
$$

where $\boldsymbol{n}_{\Gamma}$ is the outward unit normal vector of $\Gamma$.

\subsection{Definition of the potential function}

For a unified solution format of Eq. (3), the considered system is discretized into tri-node triangular elements in FDEM. A new potential function of the point $p$ in the element is defined as:

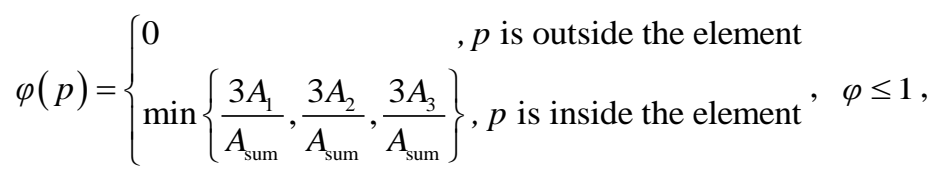

where $A_{i}, \mathrm{i}=1,2,3$ is the area of the sub-triangle, as shown in Figure 3, and $A_{\text {sum }}$ is the area of the element.

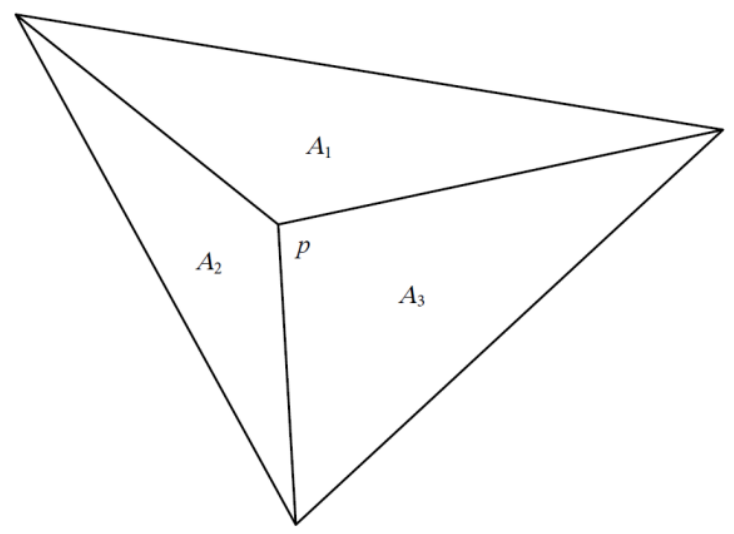

Figure 3 . The areas of the sub-triangle divided by the point $p$ in a triangle element.

Consequently, the calculation of normal contact force is carried out with Eqs. (3) and (4) by integrating the potential function over the boundaries of the overlapping area as shown in Figure 4. The simulation is simplified by managing to avoid dealing with specific various contact conditions and not having to use a rounded corner for the vertex-to-vertex contact of two triangular blocks. 


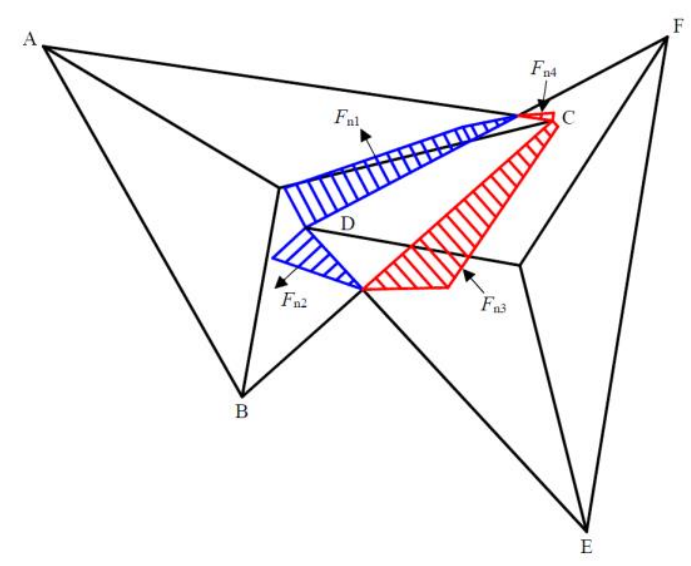

Figure 4. Normal contact force of element ABC calculated by an integration over the boundary of the penetrated area.

\section{Distance potential function}

\subsection{Some issues of contact force calculation in FDEM}

It can be clearly noticed from Eq. (3) that the potential function plays an important role in the calculation of a normal contact force and itself provides a measurement of the embeddedness between the contact couples. The potential function should achieve the same value under the same penetrated distance. In fact, as shown in Figure 5(b), despite having the same penetration distance from three bases of the sub-triangles, the equivalency of calculation results with Eq. (4) cannot be satisfied. The accuracy of Eq. (4) is limited to the condition which is shown in Figure 5(a).

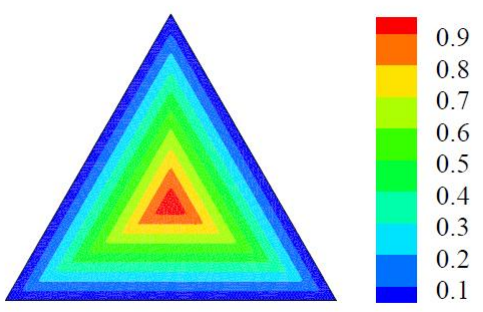

(a)

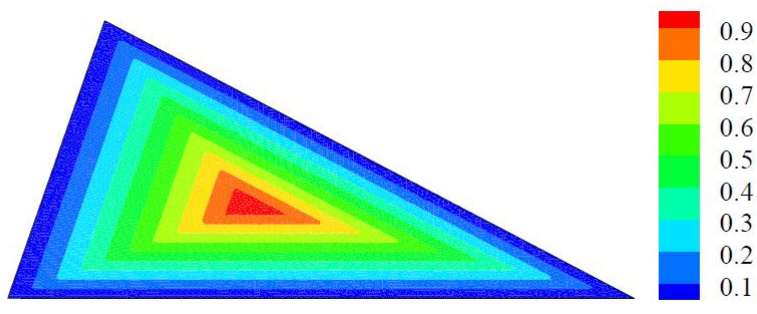

(b)

Figure 5. The distribution of potential function in triangular elements for (a) an equilateral triangle and (b) an arbitrary triangle.

The fundamental reason for this phenomenon lies in the susceptibility for the element shape. As shown in Figure 6, two same triangular elements DEF and HIJ penetrate the target element ABC (an equilateral triangle in Figure 6(a) and an arbitrary triangle in Figure 6(b)) with the same distance $h$ along the normal direction of boundary $\mathrm{BC}$ and $\mathrm{AC}$ respectively. The potential values of point $\mathrm{D}$ and $\mathrm{H}$ is given 
by Eq. (4):

$$
\varphi(\mathrm{D})=\frac{3 S_{\mathrm{DBC}}}{S_{\mathrm{ABC}}}, \varphi(\mathrm{H})=\frac{3 S_{\mathrm{HAC}}}{S_{\mathrm{ABC}}}
$$
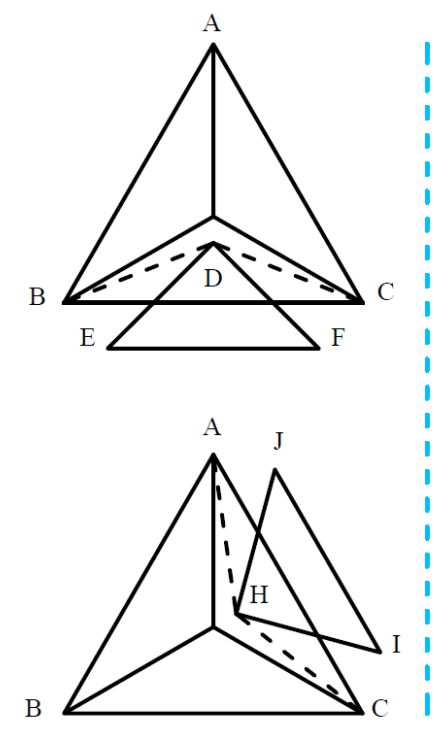

(a)
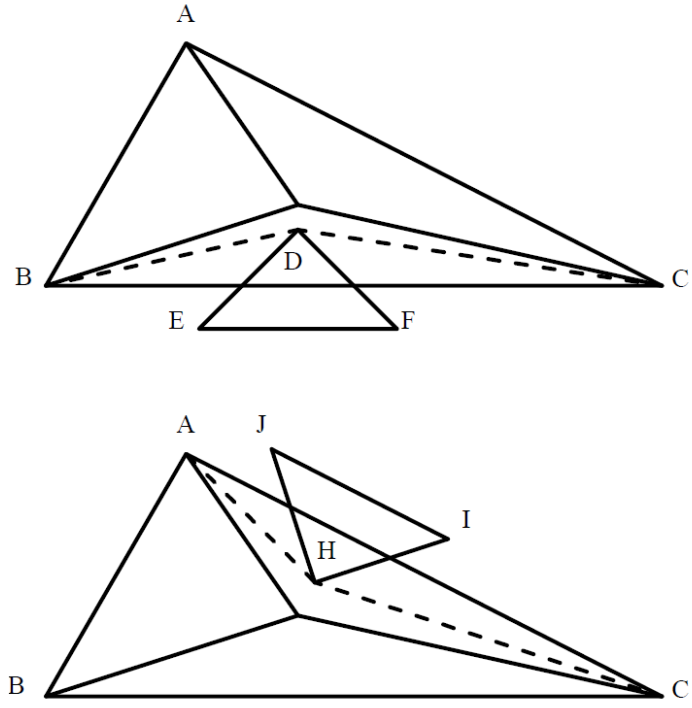

(b)

Figure 6. Two same triangular elements DEF and HIJ penetrate the target element ABC with same distance $h$ along the normal direction of boundary BC and AC respectively. (a) ABC is a regular triangle; (b) $\mathrm{ABC}$ is an arbitrary triangle.

The different shapes of triangle $\mathrm{ABC}$ obtain different results, because the triangles DBC and HAC have a large difference in the element area. In Figure $6(\mathrm{a}) \quad \varphi(\mathrm{D})=\varphi(\mathrm{H})$ because of the same area of $S_{\mathrm{DBC}}$ and $S_{\mathrm{HAC}}$. However, it presents the opposite result in Figure 6(b). The discrepancy caused by the element shape can be observed clearly. As a result, the normal contact force between the contact couples is not equivalent. However, although the element shapes have differences, the normal contact force cannot be different due to the same penetration distance in the same element.

In addition, this definition is not appropriate for polygonal elements. As shown in Figure 7(a) it can be observed that a similar distribution of potential in a rectangular element is possible. However, the equivalency of the potential values are not satisfied for points with the same penetrated distance in different sub-triangular elements. The algorithm for an arbitrary quadrilateral element can be incorrect. The presence of discontinuities along the lines between the centroid and vertex can be observed in Figure 7 (b) and the potential value is not equal to 1 at the centroid of the polygonal elements. 


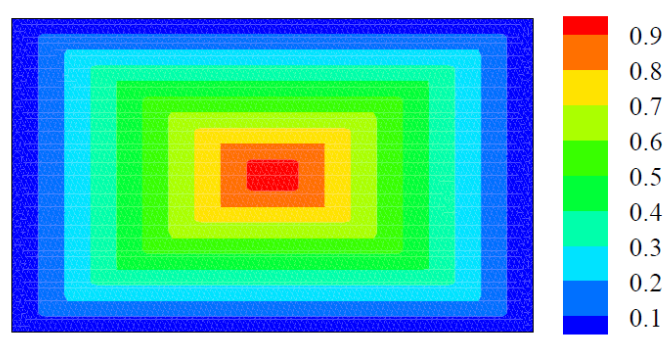

(a)

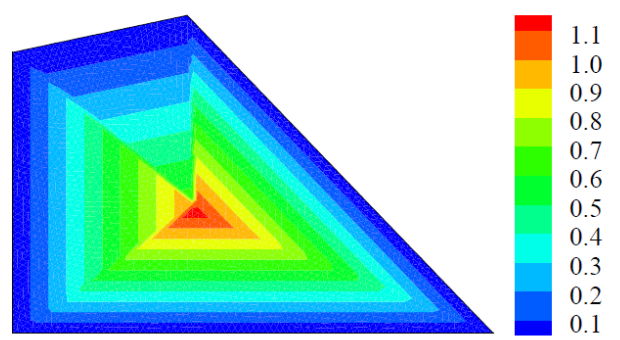

(b)

Figure 7. The distribution of potential in quadrilateral element. (a) potential distribution in a rectangle;

(b) potential distribution in an arbitrary quadrangle.

\subsection{Concept of the distance potential function}

In FDEM, a convenient and efficient approach was developed to compute the normal contact force. However, the physical meaning and numerical precision of this method remains uncertain due to the deficiency of the potential definition. In this section, a new distance potential function with a normalized partition of the embedding distance is utilized to calculate the normal contact force.

As explained in [38], the potential value should be chosen as a constant on the boundaries of elements in order to satisfy the law of conservation of energy. In this work, it is assumed that the distance potential $\varphi_{d}$ at the point $p$ inside an arbitrary polygon is defined as:

$$
\varphi_{d}(p)=\left\{\begin{array}{cc}
0, & p \text { is outside the element } \\
\frac{1}{r} h, & p \text { is inside the element }
\end{array}, \varphi_{d} \leq 1,\right.
$$

where $h$ is the distance between the point $p$ and the corresponding edge as shown in Figure 8, $r$ stands for the radius of the maximum inscribed circle of the element. Because of the arbitrary element shapes, the definition of the distance potential could result in an internal polygonal area at the center of element instead of an in-center as shown in Figure 8. The points in the polygonal area are not defined by the distance potential function. Although the distance potential singularity is observed in this polygonal region, the calculation is not affected. Considering the large value of a normal stiffness, penetration will not happen in this internal polygonal area. 


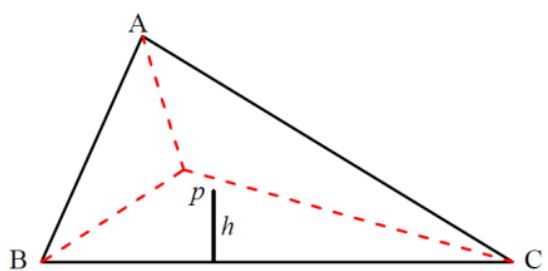

(a)

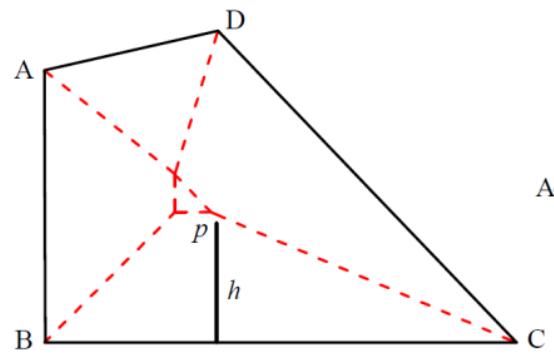

(c)

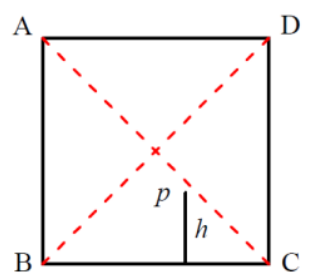

(b)

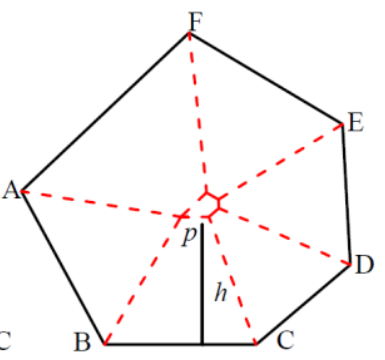

(d)

Figure 8. The distance between the point $p$ and the corresponding edge.

The merit of this definition lies in the approach of the approximated calculation of the normal contact force. Completely different from FDEM, the normal contact force is computed using the normalized partition of embedding distance instead of the original potential function. The distance potential between the contact couples has the same value for an arbitrary polygon with a fixed penetration, as shown in Figure 9. In addition, the radius of the maximum inscribed circle can also be determined in an arbitrarily convex polygon. The normal contact stiffness of different edges of the element is in accordance with the actual conditions. As a result, it can avoid errors during the calculation of the potential and the normal contact force that were discussed in section 3.1.

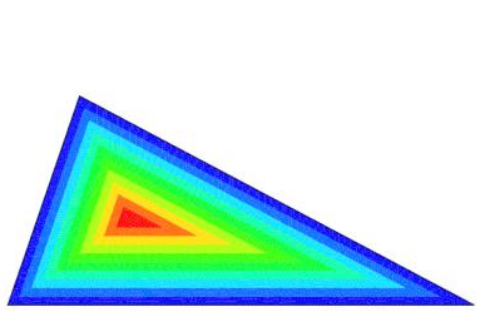

(a)

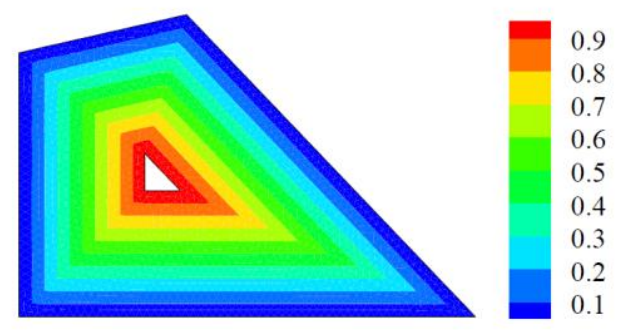

(c)

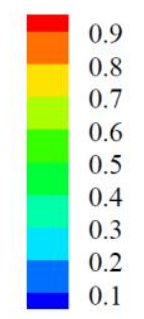

1

1
Figure 9. The distribution of the potential in polygon elements. 


\subsection{Normal contact force}

Consider two rigid convex polygonal elements in contact, as shown in Figure 10. Now by Eq. (3) and the novel distance potential definition, the normal contact force caused by the penetration of the contact element $\beta_{c}$ into the target element $\beta_{t}$ is simplified as an interaction between the element $\beta_{t}$ and the edges of $\beta_{c}$.

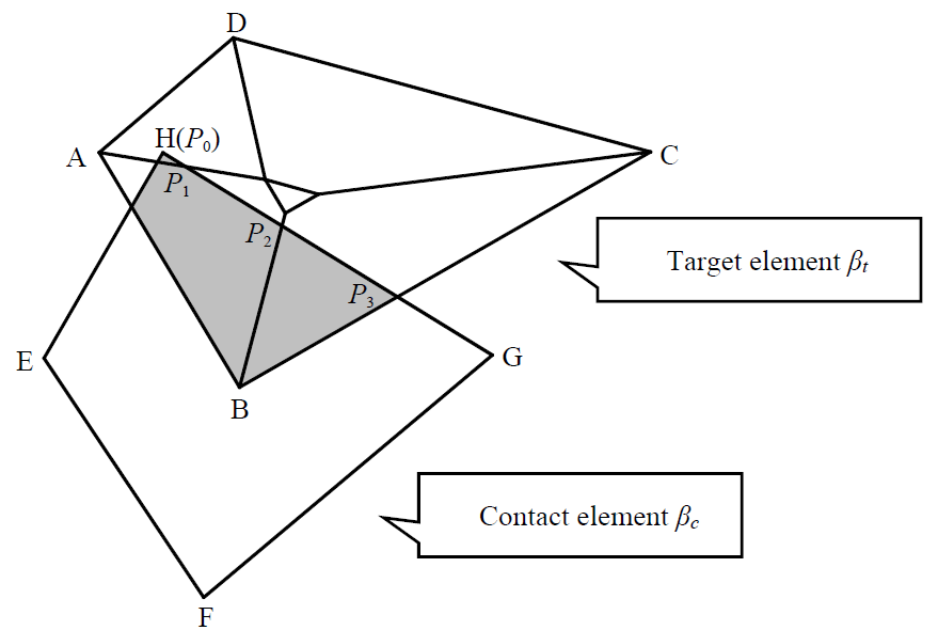

Figure 10. Contact elements and the interaction points between the element $\beta_{t}$ and the edges of $\beta_{c}$.

As presented in Figure 10, interaction points between the edge HG and the target element $\beta_{t}$ are obtained and the distance potential value for each interaction point can be calculated with Eq. (6). Then the total normal contact force on $\mathrm{HG}$ is obtained as:

$$
\boldsymbol{f}_{\mathrm{HG}, t}=\boldsymbol{n}_{\mathrm{HG}} p_{n} \int_{l_{\mathrm{HG}}}\left[0-\varphi_{d, t}(P)\right] d l=-\boldsymbol{n}_{\mathrm{HG}} p_{n} \int_{l_{\mathrm{HG}}} \varphi_{d, t}(P) d l,
$$

where $\boldsymbol{n}_{\mathrm{HG}}$ is the outward unit normal of the HG and the direction of the normal contact force, $p_{n}$ represents the penalty value, and $\varphi_{d, t}$ stands for the value of distance potential function of the point on the edge HG belonging to the element $\beta_{t}$.

It is worth to notice that the distribution of the distance potential $\varphi_{d, t}$ between the interaction points is along a straight line and linear, as shown in Figure 11. Consequently, the interaction scheme is simplified as an area calculation. 


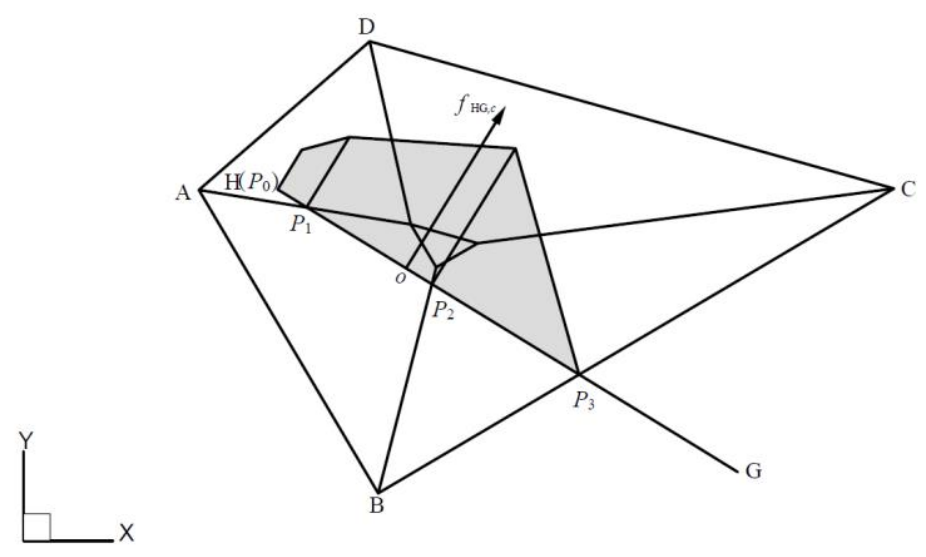

Figure 11. Distance potential distribution along the edge HG and the loading point $o$ of the total normal contact force of the edge $\mathrm{HG}$.

Thus Eq. (7) can be rewritten as:

$$
\boldsymbol{f}_{\mathrm{HG}, t}=\boldsymbol{n}_{\mathrm{HG}} p_{n} \sum_{i=0}^{n} \frac{1}{2}\left(\varphi\left(P_{i}\right)+\varphi\left(P_{i+1}\right)\right) l_{P_{i} P_{i+1}},
$$

where $l_{P_{i} P_{i+1}}$ is the distance between the interaction points $P_{i}$ and $P_{i+1}$. And the action position $o$ can be determined as the center of the potential distribution as shown in Figure 11.

The normal contact moment $\boldsymbol{M}_{\mathrm{HG}, n}$ as relative to the loading point is given:

$$
\boldsymbol{M}_{\mathrm{HG}, n}=\boldsymbol{n}_{\text {cent }-o} \times \boldsymbol{f}_{\mathrm{HG}, c},
$$

where $\boldsymbol{n}_{\text {cent }-o}$ is the vector from the contact force load position $o$ to the centroid of the element.

Accordingly, the total normal contact force $\boldsymbol{f}_{n, t}$ and the corresponding moment $\boldsymbol{M}_{n, t}$ caused by the penetration of $\beta_{c}$ to $\beta_{t}$ can be found by a repeated calculation around the remaining edges of the element $\beta_{c}$. Analogously, total normal contact force $\boldsymbol{f}_{n, c}$ and normal contact moment $\boldsymbol{M}_{n, c}$ associated with the edges of element $\beta_{t}$ caused by the penetration of $\beta_{t}$ to $\beta_{c}$ can be also obtained with Eqs. (3) and (6) as follow;

$$
\begin{gathered}
\boldsymbol{f}_{n, c}=\sum \boldsymbol{n}_{i, t} p_{n} \int\left[\varphi_{d, c}(P)\right] d l, \\
\boldsymbol{M}_{n, c}=\sum \boldsymbol{n}_{\text {cent }-i} \times \boldsymbol{f}_{i, c},
\end{gathered}
$$

where $\boldsymbol{n}_{i, t}$ is the outward unit normal of the edge $i$ of the element $\beta_{t}, \varphi_{d, c}$ represents the distance potential value of the point $p$ on the edge $i$ belonging to the element $\beta_{c}, \boldsymbol{n}_{c e n t-i}$ is the vector from the contact force load position of edge $i$ to the centroid of the element, and $\boldsymbol{f}_{i, c}$ is the normal contact force of edge $i$ caused by the penetration of $\beta_{t}$ to $\beta_{c}$.

Thus total normal contact force and moment between the contact couples is given:

$$
\begin{gathered}
\boldsymbol{f}_{n}=\boldsymbol{f}_{n, t}+\boldsymbol{f}_{n, c} \\
\boldsymbol{M}_{n}=\boldsymbol{M}_{n, t}+\boldsymbol{M}_{n, c}
\end{gathered}
$$


And the applying point of the total normal contact force is determined according to the principle of moment balance:

$$
\begin{gathered}
\boldsymbol{r}_{p n} \times \boldsymbol{f}_{n}=\boldsymbol{M}_{n}, \\
\boldsymbol{r}_{p n}=\left(x_{p n}-x_{c e n t}, y_{p n}-y_{\text {cent }}\right),
\end{gathered}
$$

where $\left(x_{p n}, y_{p n}\right)$ and $\left(x_{c e n t}, y_{\text {cent }}\right)$ are the coordinates of the applying point of the total normal contact force and the centroid of the element, respectively.

\subsection{Tangential contact force}

Another source of possible distortion of accuracy in FDEM is the omission of the tangential force. The newly proposed method takes into account the tangential contact force and a precise computational algorithm is presented on the basic idea of the force-displacement law.

In this work, the tangential contact force is obtained by following the solution of the total normal force. The direction parallels with the tangential component of the increment displacement which is decomposed according to the normal vector as shown in Figure 12. Naturally, it is perpendicular to the total normal contact force at each time step. The loading position is almost identical with the total normal contact force.

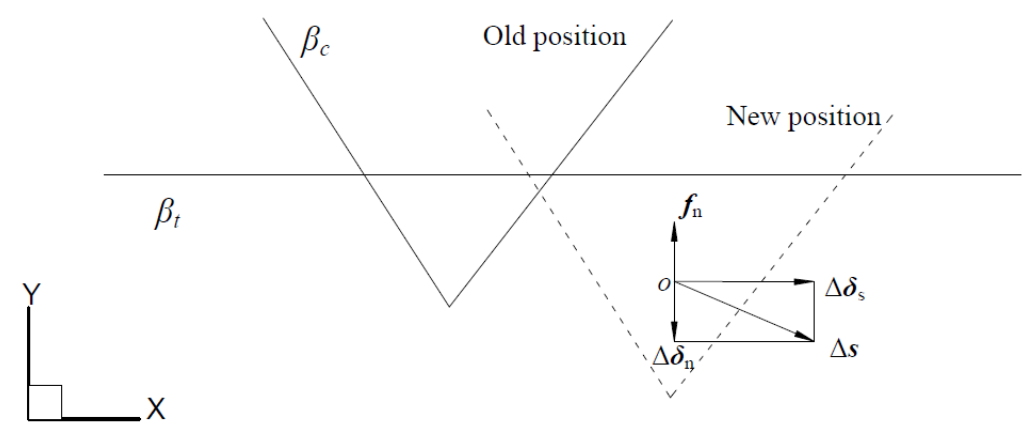

Figure 12. Tangential component of the element $\beta_{c}$.

To obtain the increment displacement, the relative velocity of the contact element $\beta_{c}$ with respect to target element $\beta_{t}$ is assumed as:

$$
v^{i}=v_{c}^{i}-v_{t}^{i}+\omega_{c}^{i} \times r_{c}^{i}-\omega_{t}^{i} \times r_{t}^{i}
$$

where $v_{c}^{i}$ and $v_{t}^{i}$ are the translational velocities of blocks $\beta_{c}$ and $\beta_{t}$ at step $i$, respectively; $\omega_{c}^{i}$ and $\omega_{t}^{i}$ stand for the angular velocities of blocks $\beta_{c}$ and $\beta_{t}$, respectively. Then the incremental tangential displacement $\Delta \boldsymbol{\delta}_{s}^{i}$ is:

$$
\Delta \boldsymbol{\delta}_{s}^{i}=\Delta \boldsymbol{s}^{i}-\left(\Delta \boldsymbol{s}^{i} \cdot \boldsymbol{n}_{n}\right) \cdot \boldsymbol{n}_{n}=\left[\boldsymbol{v}^{i}-\left(\boldsymbol{v}^{i} \cdot \boldsymbol{n}_{n}\right) \cdot \boldsymbol{n}_{n}\right] \cdot \Delta t
$$


The tangential contact force is updated as:

$$
f_{s}^{i}=r \times f_{s}^{i-1}+\Delta f_{s}^{i}
$$

where $r$ is the rotation matrix that rotates the normal vector from step $i$ - 1 to the normal vector at the current step $i, \quad r=\left[\begin{array}{cc}n_{2} & -n_{1} \\ n_{1} & n_{2}\end{array}\right]^{T} \times\left[\begin{array}{cc}n_{2}^{\prime} & -n_{1}^{\prime} \\ n_{1}^{\prime} & n_{2}^{\prime}\end{array}\right]$, in which $\left(n_{1}, n_{2}\right)$ and $\left(n_{1}^{\prime}, n_{2}^{\prime}\right)$ are the unit normal direction vector at the step $i$ and $i$-1, respectively, $\Delta f_{s}^{i}$ is the incremental tangential contact force expressed as:

$$
\Delta \boldsymbol{f}_{s}^{i}=k_{s} \cdot \Delta \boldsymbol{\delta}_{s}^{i}
$$

The magnitude of the tangential force is checked with the maximum possible value $\left(f_{s}\right)_{\max }^{i}$ defined by the Coulomb-type friction law:

$$
\left(f_{s}\right)_{\max }^{i}=f_{n}^{i} \cdot \tan \varphi_{\mu}+c
$$

where $\varphi_{\mu}$ is the maximum static friction angle, $c$ represents the cohesion force, $f_{n}^{i}$ stands for the magnitude of the total normal contact force at step $i$. If the absolute value of $f_{s}^{i}$ is larger than $\left(f_{s}\right)_{\max }^{i}$, $f_{s}^{i}$ is set to be equal to $\left(f_{s}\right)_{\max }^{i}$.

The tangential contact moment is given by:

$$
\boldsymbol{M}_{s}^{i}=\boldsymbol{n}_{\text {cent }-s}^{i} \times \boldsymbol{f}_{s}^{i},
$$

where $\boldsymbol{M}_{s}^{i}$ is the tangential contact moment at step $i$, and $\boldsymbol{n}_{\text {cent-s }}^{i}$ is the vector from the force load position to the centroid of the element at step $i$.

\section{Contact detection algorithm}

The contact detection algorithm for the discrete element method must be robust and efficient in order to reduce the requirement of CPU and computer memory, because the total cost of the processing of contact interactions for all possible contact couples is proportional to $N^{2}$, where $N$ is the total number of discrete elements. Large-scale discrete element models always involve substantial individual blocks and thus the computational cost of DEM can be largely affected by the contact algorithm. To address this issue, Munjiza [53] proposed a linear contact detection algorithm, the no binary search (NBS) algorithm, in which both the total CPU time and computer memory requirements are proportional to the total number of the blocks. However, the NBS algorithm is based on a strict condition of the element size and it cannot be applied to solve the problems that include non-uniform blocks. The calculation area is discretized according to the largest element, and as a consequence, contact detections for the small blocks are also performed even when they are far away from each other. It greatly degrades the calculating efficiency. Subsequently, Munjiza [61] developed the multi-step Munjiza-Rougier algorithm (MMR) for particles 
with different sizes.

In this work, a new contact detection algorithm is developed for non-uniform blocks based on the square bounding box by combining the NBS and MMR algorithm.

The basic idea for this algorithm is dividing the blocks into groups according to the size of the element:

Group 1: size of bounding box for the first group blocks $d_{1}: d_{1}=D_{\max }$;

Group 2: size of bounding box for the second group blocks $d_{2}: d_{2}=D_{\max } / \alpha, \alpha>1$;

Group 3: size of bounding box for the third group blocks $d_{3}: d_{3}=D_{\max } / \alpha^{2}, \alpha>1$;

Group $n$ : size of bounding box for the n group blocks $d_{\mathrm{n}}: d_{n}=D_{\max } / \alpha^{n-1}, \alpha>1$, where $D_{\max }$ is the maximum circumcircle diameter among all the blocks.

Then the process of this contact detection algorithm can be performed in $n$ steps:

Step 1: All the elements are mapped into the space which is divided into cells based on $d_{1}$ as shown in Figure 13. According to the current positions of the centroid of elements each block can be mapped to only one cell following the formulation:

$$
\begin{aligned}
& x_{k}=1+\operatorname{int}\left(\frac{x_{i}-x_{\min }}{l_{\max }}+0.5\right), \\
& y_{k}=1+\operatorname{int}\left(\frac{y_{i}-y_{\min }}{l_{\text {max }}}+0.5\right),
\end{aligned}
$$

where $x_{i}, y_{i}$ are the coordinates of the centroids in global coordinate system. $x_{k}, y_{k}$ are the coordinates of the centroids when the elements are mapped into the cells, and $l_{\max }$ is the size of the cell at each step.

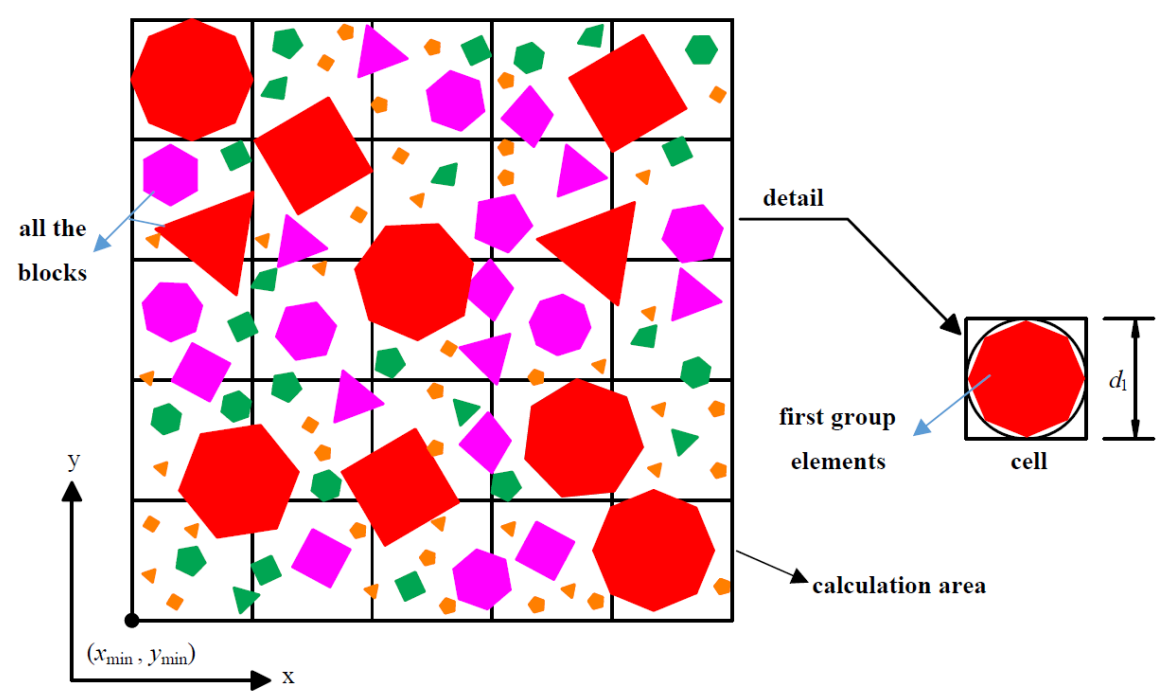

Figure 13. Illustration of the new non-uniform block contact algorithm, where blocks are divided into four groups based on the element size and space divided into identical cells which are large enough to contain the first group element. 
Then, contact detection is performed for the first group element. The detection of contact for the first group element is circulated as a direct check and the discrete elements are regarded as either central or neighboring cells, as shown in Figure 14. Two discrete elements mapped into the cells can be determined as contact couples if the contact condition is met:

$$
d \leq l,
$$

where $d$ is the distance between the centroids of the contact couples, and $l$ is the contact distance: $l=l_{1 \max }+l_{2 \max }$, where $l_{1 \max }, l_{2 \max }$ are the longest distances between the vertexes and centroids of the two contact elements, respectively.

The contact elements for the first group elements can be obtained respectively.

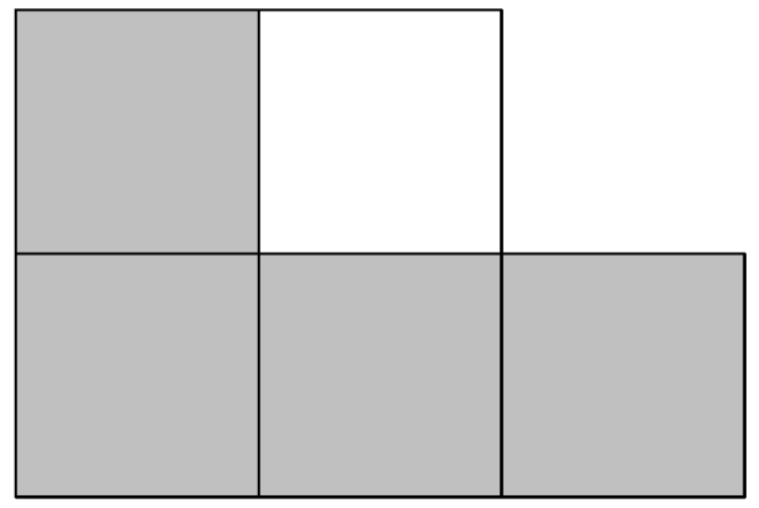

Figure 14. Contact check cells illustration, where the blank cell is the central cell and the shaded elements are the neighboring cells.

Step 2: The first group elements are removed from the system and all the remaining elements are mapped into the space which is divided using $d_{2}$ as shown in Figure 15. Then contact detection is performed between the elements of group 2 and the remaining group elements with the same method as in step 1.

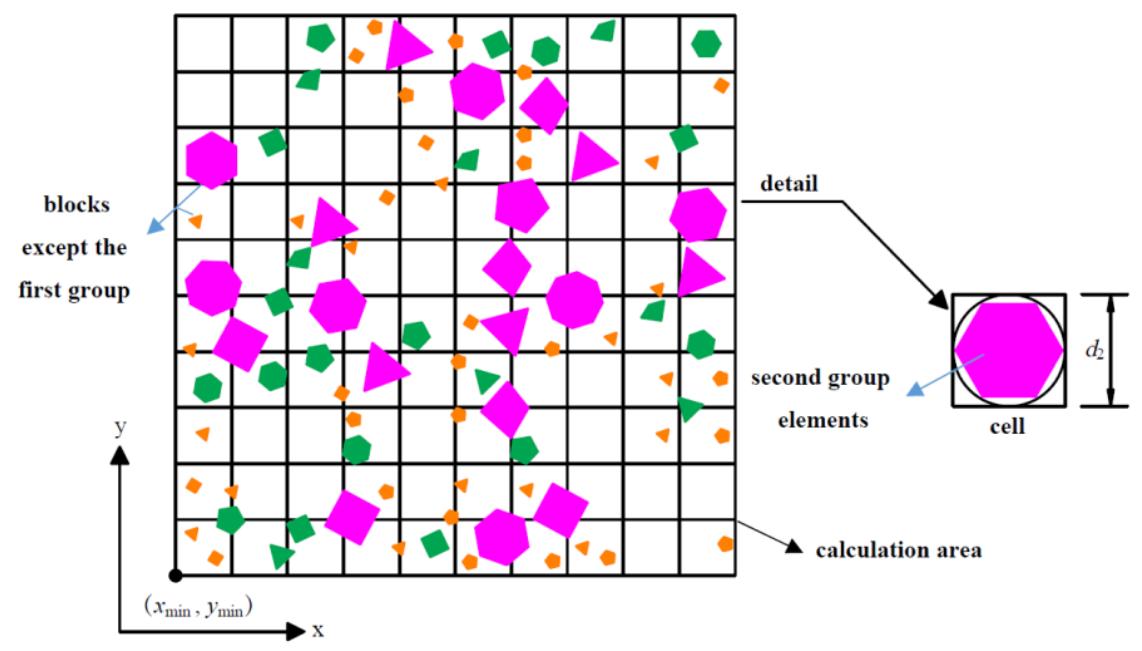


Figure 15. Step 2 illustration, where the first group elements are removed and space is divided into identical cells using $d_{2}$ as the length scale.

Step $n$ : By analogy, in this step only elements in group $n$ remain in the system as shown in Figure 16 and the contact detection is carried out between those elements.

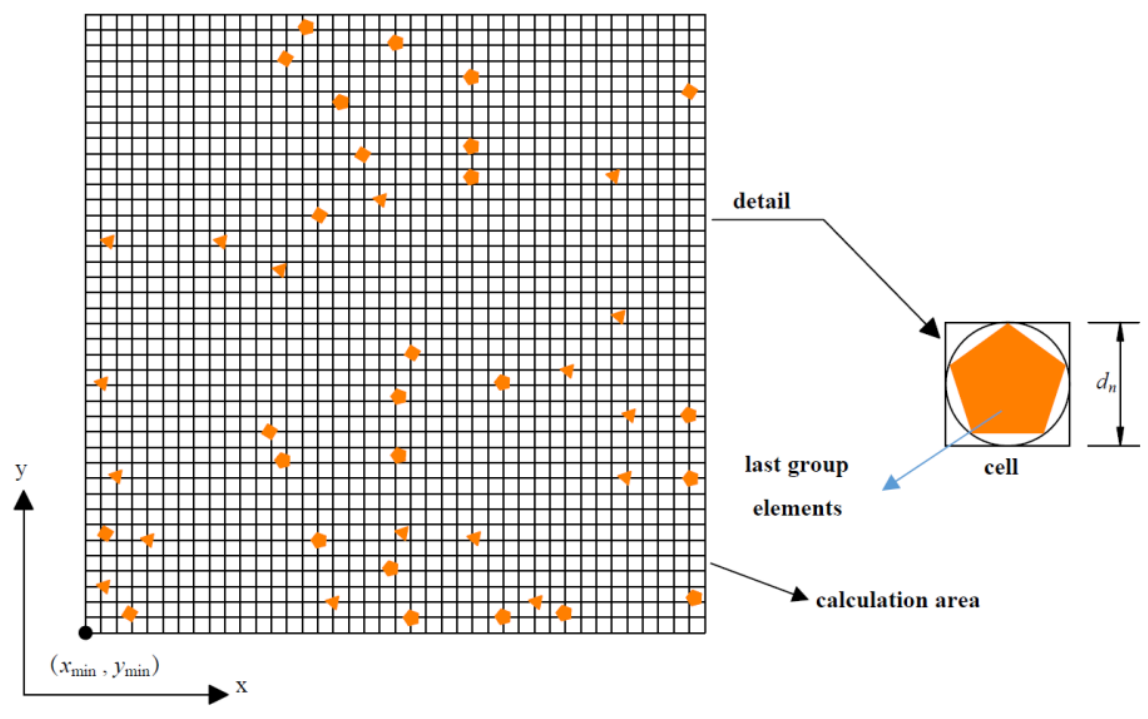

Figure 16. Contact detection for the last group elements.

With the new contact detection algorithm, the size of the cell in each step is approximately equal to that of the detection elements. The coordinates of the elements mapped into the cells show the actual distribution law of the elements and thus greatly improve the accuracy of contact detection for elements with different sizes.

\section{Numerical examples}

Several numerical examples are presented in this section to provide validation of the accuracy and capability for this newly proposed method. Firstly, the reliability and accuracy in the normal contact force calculation is verified by simulating the impact process between triangular blocks. Then energy conservation property is proved by a test using a beam-shaped heap of rigid fragments. A numerical frictional experiment composed of two blocks is simulated to explain the ability in tracking the friction interaction. Another benchmark is about the block accumulation which illustrates the computational efficiency for arbitrary polygonal elements of this method. Then the method is applied to model the sliding/toppling test for rock slope and the joints structure effects in the sliding rock mass. Finally the simulation of Tangjiashan landslide is performed to illustrate the capability of this proposed method to deal with a practical problem of large scale. 


\subsection{Simulation of impact between triangular blocks}

As already noted, the proposed method can provide an accurate normal contact force without the influence of the element shape. In this example, a numerical simulation is carried out to verify this assertion

The problem consists of two triangular blocks A and B which are fixed on a horizontal plane, and block C falls towards A and B due to gravity. The shape and size of blocks A and B are the same and the geometry for this problem is illustrated in Figure 17. The density of the blocks is $2000 \mathrm{~kg} / \mathrm{m}^{3}$ and the initial velocity of block $\mathrm{C}$ is set as $0 \mathrm{~m} / \mathrm{s}$. The vertical acceleration due to the gravity is $-10 \mathrm{~m} / \mathrm{s}^{2}$, friction is neglected. In this simulation, large embedding is allowed with the application of a small contact stiffness for observation of the process of penetration.

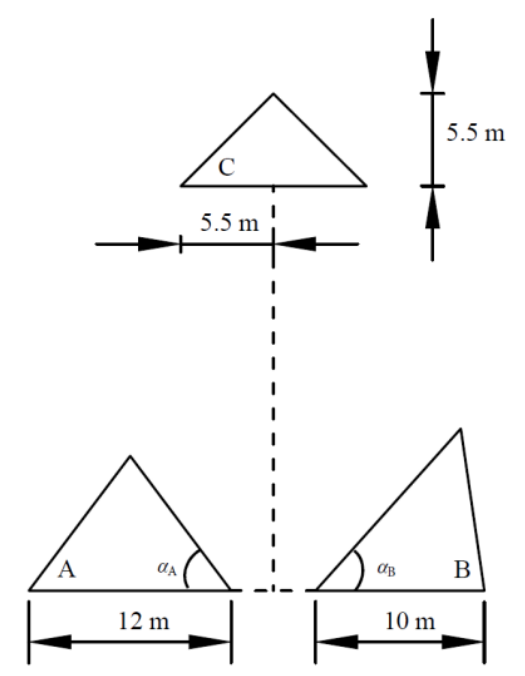

Figure 17. Numerical model of the impact test.

Initially block $\mathrm{C}$ is free-falling towards $\mathrm{A}$ and $\mathrm{B}$, and then turns into a reverse movement after the impact with blocks $\mathrm{A}$ and $\mathrm{B}$ due to the asymmetrical contact force caused by the penetrations between the contact couples A, C and B, C. The reason is that A and B have the same geometry, same physical parameters, the same angle between the inclined surfaces and the horizontal plane $\alpha_{\mathrm{A}}$ and $\alpha_{\mathrm{B}}$, and more importantly the same penetration between the two contact couples.

The predicted motion of block $\mathrm{C}$ is given in Figure 18. From these results, it can be clearly observed that block $\mathrm{C}$ falls down initially and then bounces along the original falling trajectory. The simulation with the proposed method follows the expected motion of rigid body impact. 


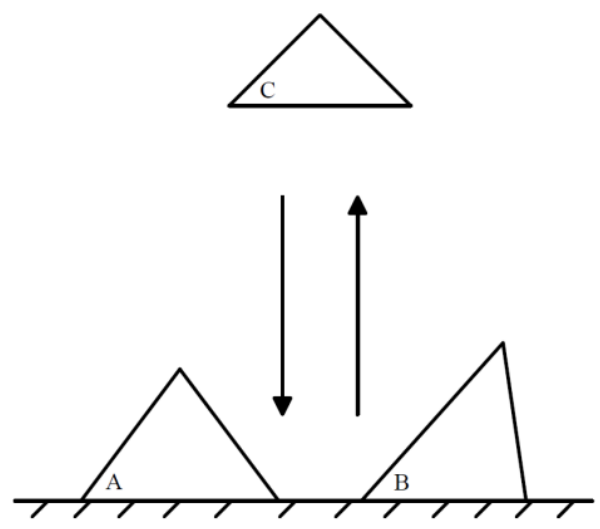

Figure 18. Predicted motion block $\mathrm{C}$ simulated by the proposed method.

For comparison, the result achieved with FDEM is exhibited in Figure 19. It is worthwhile to notice that the motion trajectory is not the same before and after the collision and a horizontal movement and deflection of block $\mathrm{C}$ is observed in the simulation. The trajectories of block $\mathrm{C}$ obtained by the two methods are compared with each other as shown in Figure 20(a). As explained in section 3.1, the normal contact force between the two contact couples can be unequal with each other in FDEM as defined by the potential function as described in Figure 21(b). This is because the potential function in FDEM is not strictly a function of the penetration distance. It results in a horizontal displacement of block $\mathrm{C}$ after it impacts with the blocks A and B as shown in Figure 20(b).

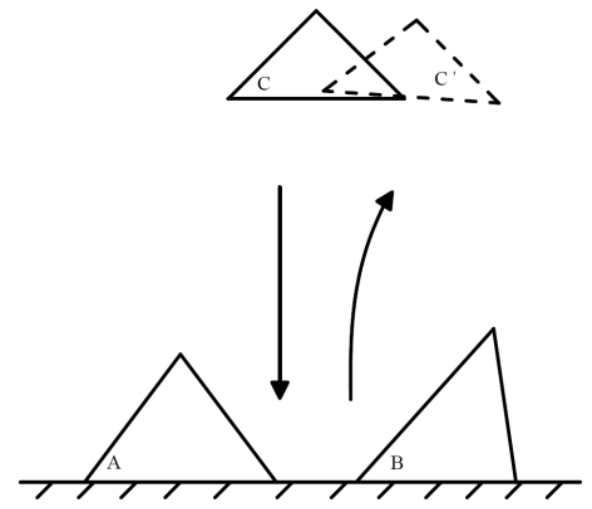

Figure 19. Predicted motion block C simulated by FDEM. 


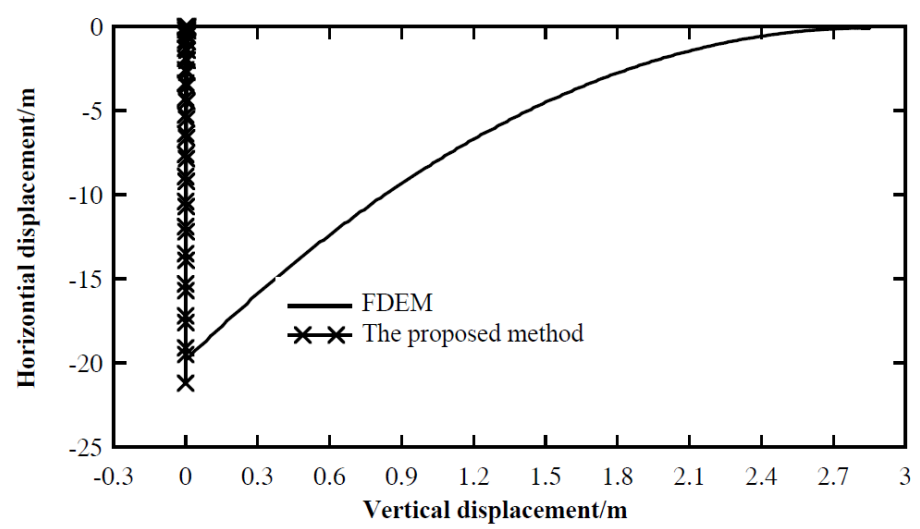

(a)

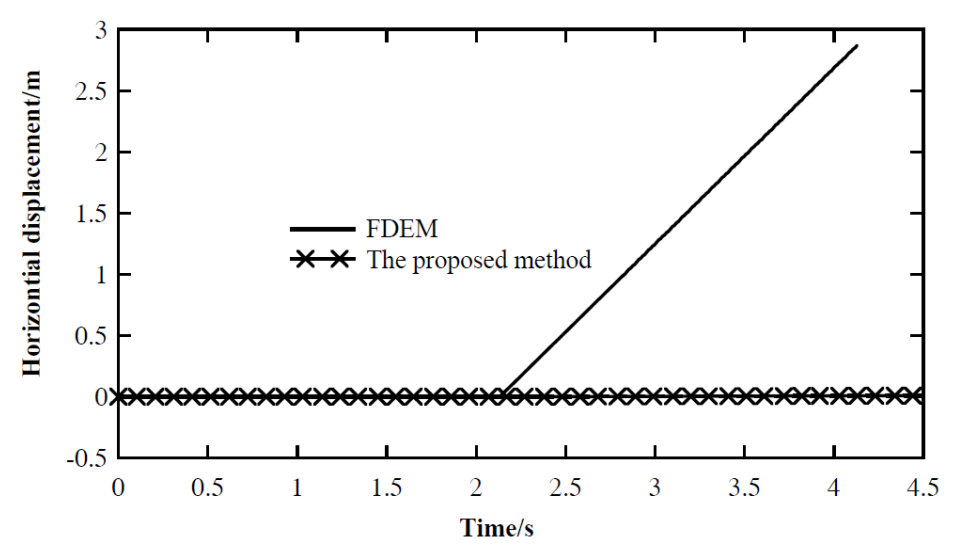

(b)

Figure 20. Simulation results by two methods for the impact case of triangular blocks, where (a) the motion trajectories of block $\mathrm{C}$ is shown by two methods, and (b) the time variation of the horizontal displacement of block $\mathrm{C}$.

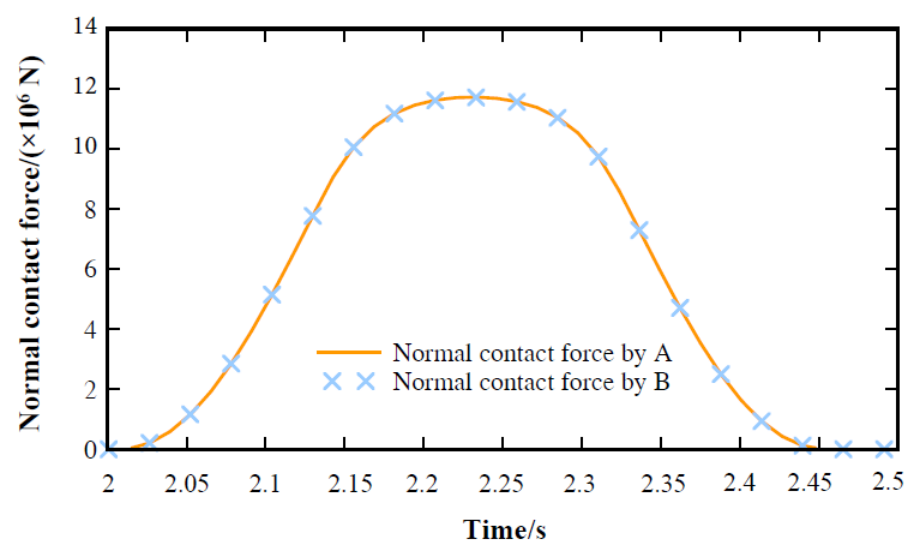

(a) 


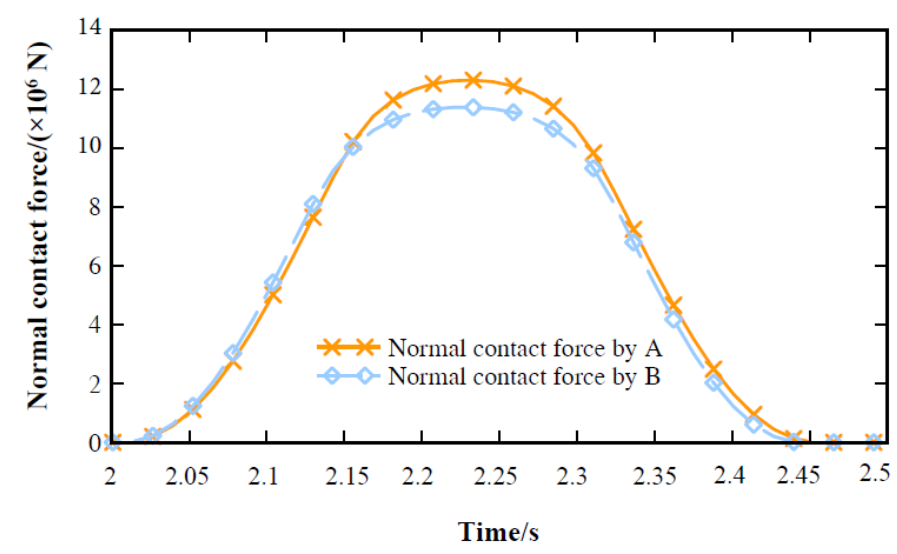

(b)

Figure 21. Normal contact force between the contact couples A, C and B, C obtained by two methods for the impact case of triangular blocks, where (a) the normal contact force calculated by the proposed method, and (b) the normal contact force calculated by FDEM.

\subsection{Beam-shaped heap of rigid fragments}

In order to evaluate the energy conservation and computational stability of the proposed method, the simulation of beam-shaped heap of rigid fragments is simulated following Munjiza [38]. The original shape of the heap is shown in Figure 22. The model is discretized by $16 \times 160$ triangle elements. The velocity of the rigid projectile is set as constant with $u=-50 \mathrm{~m} / \mathrm{s}$ as shown in Figure 22. For comparison, the test with the same setting is also simulated by FDEM.

The predicted transient motion of the heap is given in Figure 23. Both the proposed method and FDEM present the same relative motion trend. Initially the fragments are stacked each other that they contact, however there is no overlap or contact force between the fragments. As the projectile impacts the center of the heap, the fragments in heap near the impact point are extracted by the projectile. This result is an acceleration of these fragments while moving at the opposite direction of the triangular projectile. Due to the interaction between the fragments, the disturbance spreads from the initial impact point to the edges of the heap, causing further fragments to move away from each other. The result is that the shape of the heap begins to change and the packing density decreases.

When the projectile finally goes through the heap, the shape and the density of the heap have considerably changed. At this stage, the fragments and projectile separate with each other in a constant velocity. It is worth to notice that the symmetry of the heap is preserved during the whole process of the impact.

Figure 24 shows the evolution of the energy conservation obtained by the proposed method and FDEM. The reduction of the kinetic energy is a consequence of the contact. The kinetic energy translates into the potential energy due to the overlapping of the fragments in contact. Then the potential energy is 
zero and the kinetic energy is equal to the initial value, when the fragments and projectile get a constant velocity.
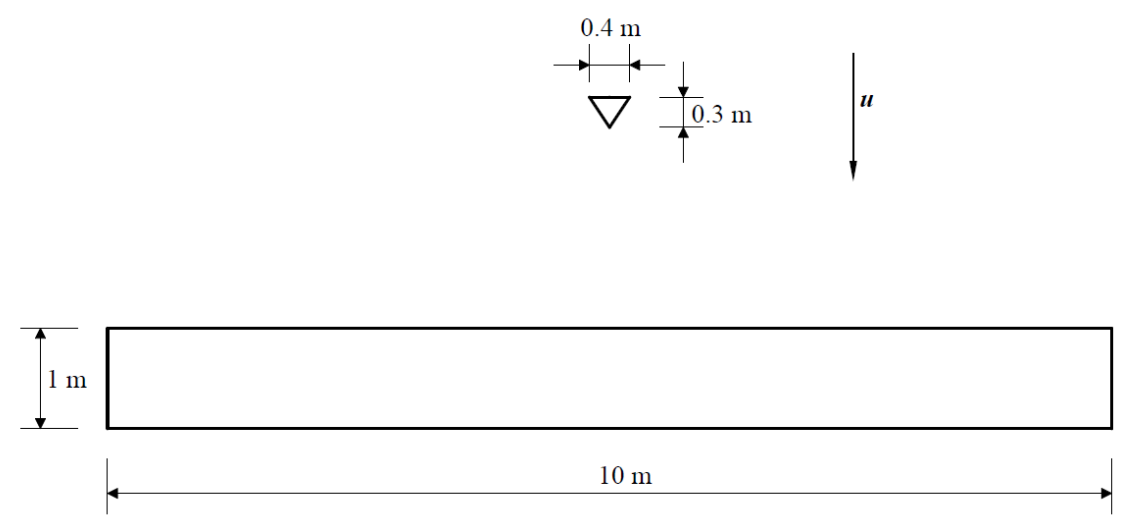

Figure 22. Model of the beam-shaped heap.

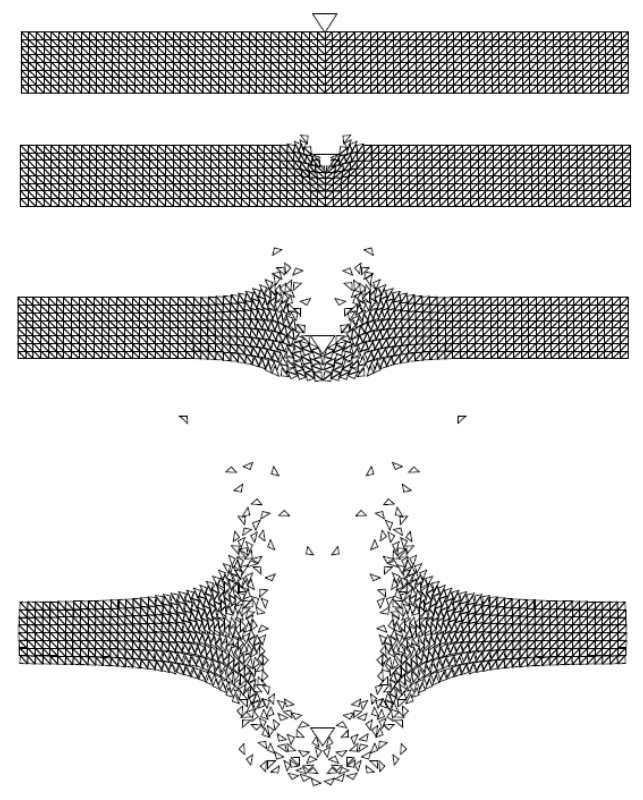

(a)
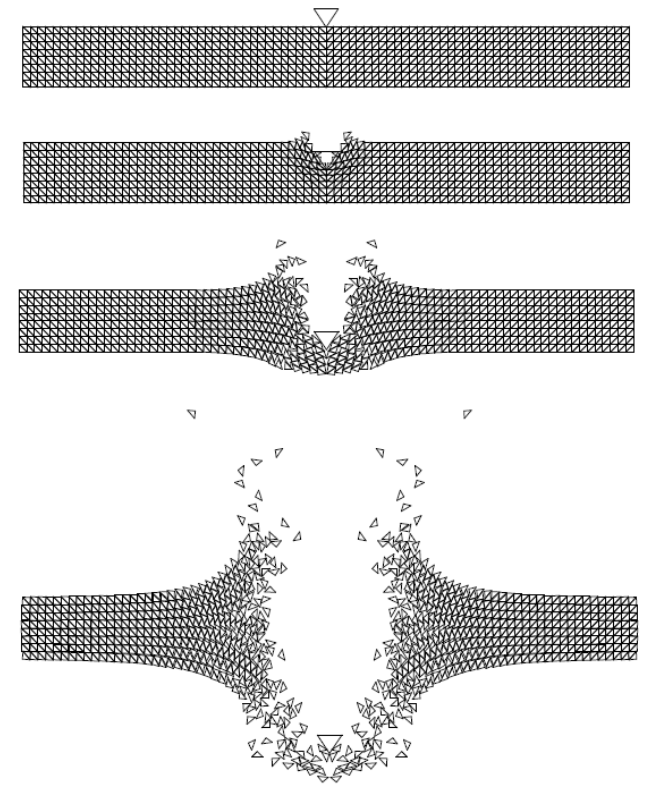

(b)

Figure 23. Predicted transient motions of the fragments; (a) the simulation result by the proposed method; (b) the simulation result by FDEM. 


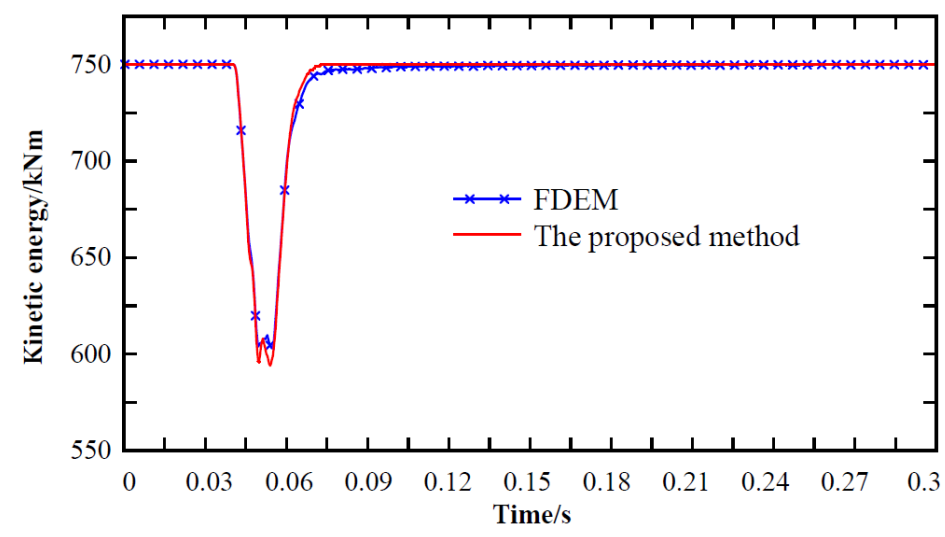

Figure 24. Kinetic energy as a function of time calculated by two methods.

\subsection{Test for the numerical frictional experiment}

In this example, the process of a quadrate block sliding on a horizontal surface with an initial horizontal velocity is simulated as a benchmark test of a friction problem for this proposed method.

Table 1 lists the geometric information and material properties of the sliding quadrate block for this test.

Table 1

Geometric information and material properties of two sliding quadrate blocks for this simulation.

\begin{tabular}{cccccc}
\hline Parameter & Length $(\mathrm{m})$ & $\begin{array}{c}\text { Density } \\
\left(\mathrm{kg} / \mathrm{m}^{3}\right)\end{array}$ & $\begin{array}{c}\text { Young's } \\
\text { modulus }(\mathrm{Pa})\end{array}$ & $\begin{array}{c}\text { Normal } \\
\text { stiffness } \\
(\mathrm{N} / \mathrm{m})\end{array}$ & $\begin{array}{c}\text { Tangential } \\
\text { stiffness } \\
(\mathrm{N} / \mathrm{m})\end{array}$ \\
\hline Value & 0.5 & 2600 & $1 \times 10^{9}$ & $1.5 \times 10^{9}$ & $1.2 \times 10^{9}$ \\
\hline
\end{tabular}

Figure 25 presents the simulation model of this test. The block is given an initial horizontal velocity $u=4 \mathrm{~m} / \mathrm{s}$. The horizontal velocity decreases linearly because of the influence of friction until it stops at a distance that can be analytically calculated.

Figure 26 and 27 show the displacement and velocity of this block with a constant friction coefficient $\left(\varphi_{\mu}\right)$ 0.1, 0.16 and 0.2 using the proposed method and theoretical analysis, respectively. The results show perfect agreement between the proposed method and the theoretical results.

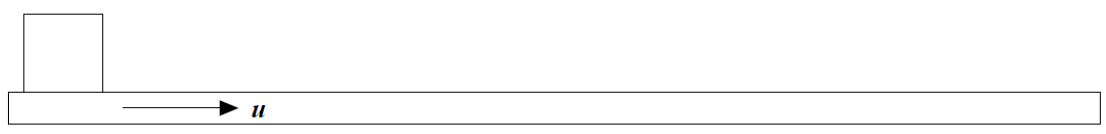

Figure 25. Model of the numerical friction experiment. 


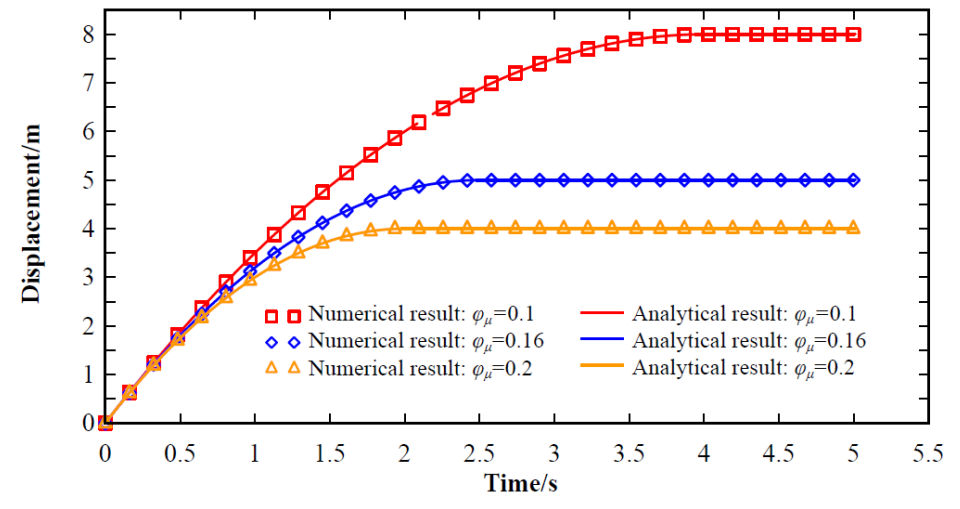

Figure 26. Time variation of the displacement of the sliding quadrate block for several friction coefficients as calculated by the proposed numerical method and analytically.

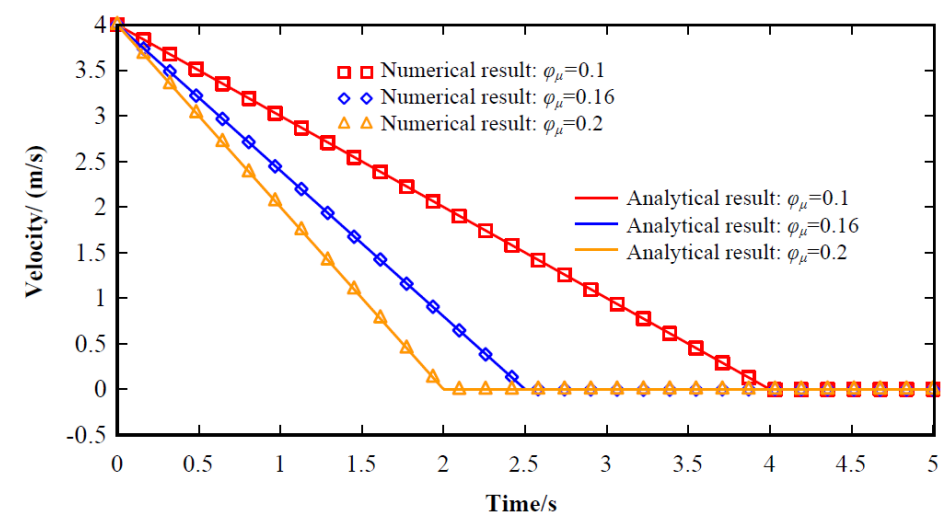

Figure 27. Time variation of the velocity of the sliding quadrate block for several friction coefficients as calculated by the proposed numerical method and analytically.

Figure 28 shows the results of displacement time variation for several values of the normal stiffness. Again, an excellent match is revealed between the proposed method and the analytical results.

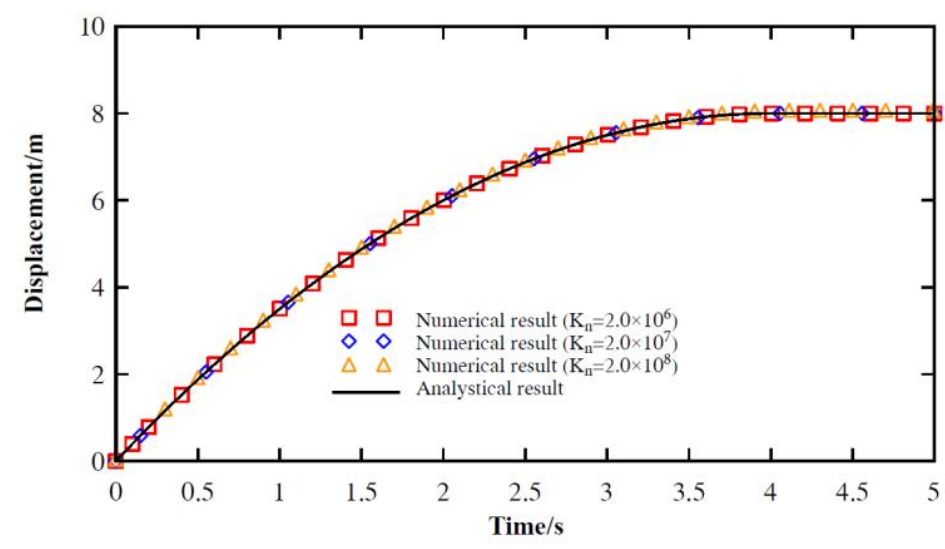

Figure 28. Time variation of the displacement of the sliding quadrate block for several values of the normal stiffness as calculated by the proposed numerical method and analytically. 


\subsection{Simulation of block accumulation}

The following example is used to validate the ability of the proposed method to calculate the motion of arbitrary convex elements.

To simulate the process of deposition under the influence of gravity, a rigid box of dimension 32 $\mathrm{mm} \times 122 \mathrm{~mm}$ is adopted. Four series of blocks with different shapes fall into the box and achieve a stable state by applying the same friction coefficient of block-block and block-wall, $\varphi_{\mu}=0.15$ and a damping ratio 0.002. The element models used in this example are presented in Figure 29.The material properties of all the blocks are assumed to be identical as described in Table 2:

Table 2

Material properties of the blocks.

\begin{tabular}{|c|c|c|c|c|c|c|}
\hline Parameter & $\begin{array}{c}\text { Total } \\
\text { volume of } \\
\text { blocks } \\
\left(\mathrm{mm}^{2}\right)\end{array}$ & $\begin{array}{l}\text { Density } \\
\left(\mathrm{kg} / \mathrm{m}^{3}\right)\end{array}$ & $\begin{array}{l}\text { Young's } \\
\text { modulus } \\
(\mathrm{Pa})\end{array}$ & $\begin{array}{c}\text { Poisson } \\
\text { radio }\end{array}$ & $\begin{array}{c}\text { Normal } \\
\text { stiffness } \\
(\mathrm{N} / \mathrm{m})\end{array}$ & $\begin{array}{c}\text { Tangential } \\
\text { stiffness } \\
(\mathrm{N} / \mathrm{m})\end{array}$ \\
\hline Value & 800 & 2000 & $1 \times 10^{9}$ & 0.167 & $2 \times 10^{9}$ & $2 \times 10^{9}$ \\
\hline
\end{tabular}

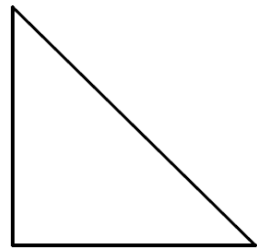

(a)

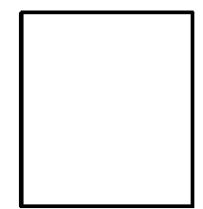

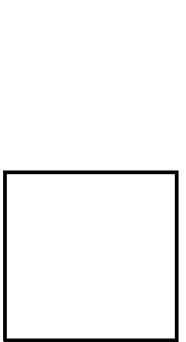

(b)

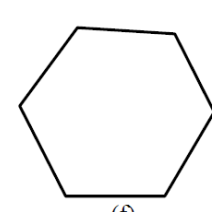

(f)

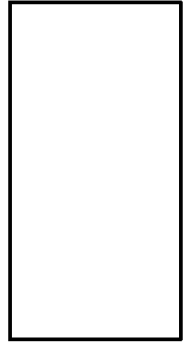

(c)

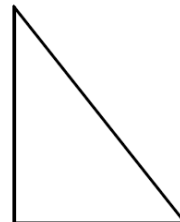

(d)
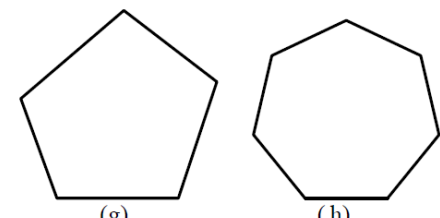

Figure 29. Simulation models of four series of deposition consisting of (a) triangles; (b) quadrates; (c) rectangles; and (d)-(h) all five different element types are in the fourth set of simulations.

The simulation results can be seen in Figure 30. For comparison, the same example is also simulated with FDEM in which the polygonal blocks are represented by the triangular blocks bundled together with springs as shown in Figure 31. The simulation methods achieve similar results exhibited in Figure 32 and both two methods reflect the block motion. However, two approaches present different performance when dealing with this problem of polygonal elements. Table 3 lists the total calculation time of the two methods for the polygonal elements. Clearly, the proposed method performs much better and it is much more suitable and efficient for the medium involves arbitrary polygonal elements than the FDEM. 


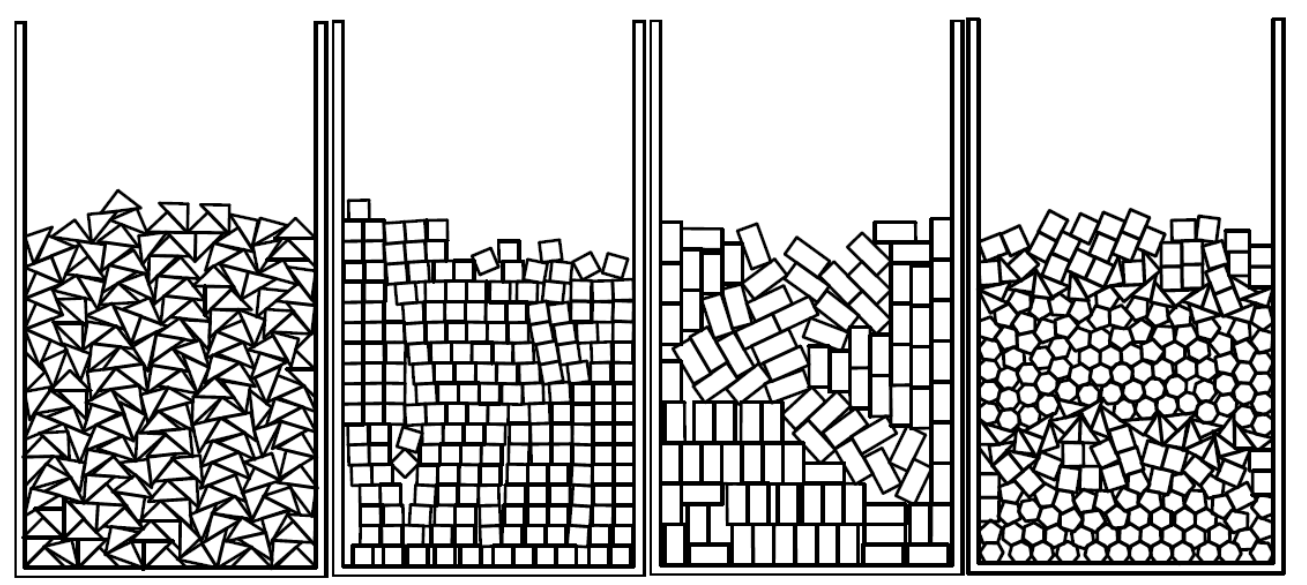

(a)

(b)

(c)

(d)

Figure 30. Simulation results of the four series of deposition examples with the proposed method and for elements of (a) triangles; (b) quadrates; (c) rectangles; and (d) mixed shapes.

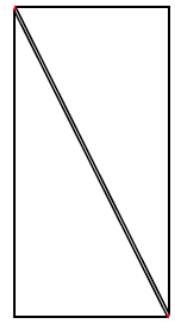

(a)

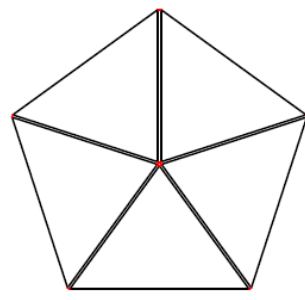

(b)

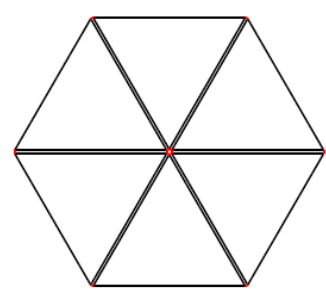

(c)

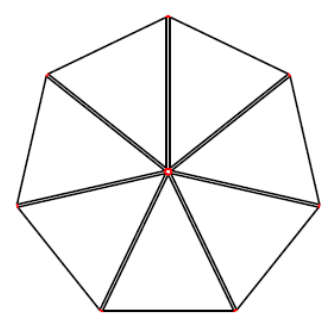

(d)

Figure 31. The element types of several triangular elements bundled together with springs which have a high elastic stiffness for the FDEM simulation: (a) a quadrangle-shape element; (b) a pentagon-shape element; (c) a hexagon-shape element; and (d) a heptagon-shape element.

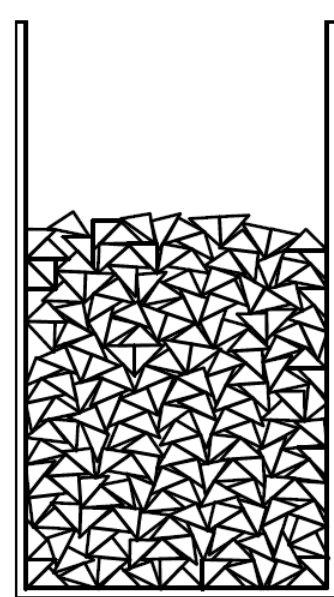

(a)

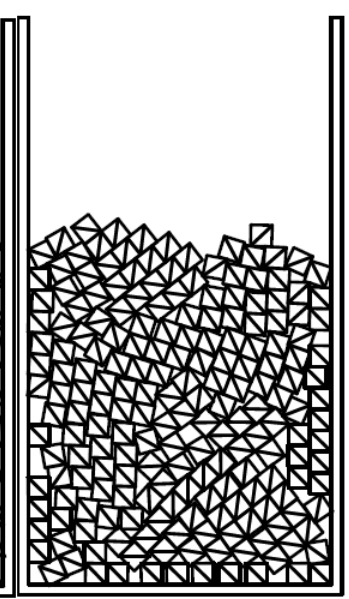

(b)

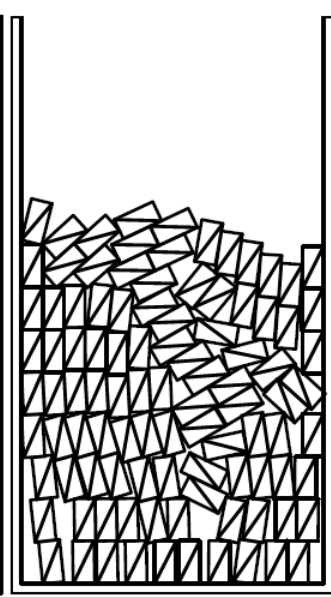

(c)

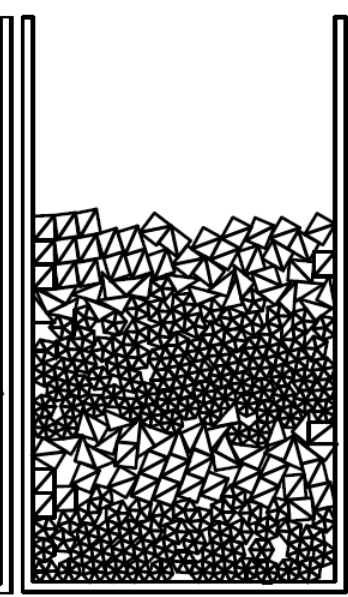

(d) 
Figure 32. Simulation results of the four series of deposition examples by FDEM and for the elements of (a) triangles; (b) quadrates; (c) rectangles; and (d) mixed shapes.

\section{Table 3}

The computer time required by two methods in simulating the accumulation of blocks with different shapes.

\begin{tabular}{ccl}
\hline & The proposed method & FDEM \\
\cline { 2 - 3 } Element type & & \\
& Time (s) & Time (s) \\
\hline Quadrate & & 1825.9 \\
\hline Rectangle & 759.4 & 1284.5 \\
\hline Mixed & 594.3 & 2760.3 \\
\hline
\end{tabular}

\subsection{Sliding/toppling test of a joint rock slope}

The limit equilibrium analysis of toppling failure for a joint slope described by Goodman and Bray [62] is a popular validation case for various discontinuous methods because of the relatively simple geometry, boundary conditions and conditions of external loads. Heok and Bray [63] give an example of a toppling problem of two dimensional joint slope with the limit equilibrium analysis method (Figure 34). In this paper, this example is adopted to test the proposed method by comparing the numerical results with the analysis results provided by Heok and Bray.

In this example, a rock slope of $92.5 \mathrm{~m}$ height which is cut at an angle of $56.6^{\circ}$ in a layered rock mass dipping at $60^{\circ}$ into the base of the block. The slope is discretized into 16 blocks which are considered as rigid and rested on a base stepped at $1 \mathrm{~m}$ and the block 10 is at the crest. The width of each block $\Delta x$ is $10 \mathrm{~m}$, and the height and the ratio between the height and width $y_{n} / \Delta x$ of each block are shown on Table 4. The friction angle between the faces and bases of the blocks is set to be $38.15^{\circ}\left(\phi_{f}\right)$ and the unit weight of the rock is $25 \mathrm{kN} / \mathrm{m}^{3}$. The geometry of the simulation is shown in Figure 34 .

Table 4

Geometric information of the blocks for this simulation, where $y_{n}$ and $\Delta x$ are defined in Figure 33.

\begin{tabular}{cccc}
\hline$n$ & $y_{n}$ & $\Delta x$ & $y_{n} / \Delta x$ \\
\hline 1 & 4.0 & 10 & 0.4 \\
\hline 2 & 8.0 & 10 & 0.8 \\
\hline 4 & 12.0 & 10 & 1.2 \\
\hline 5 & 16.0 & 10 & 1.6 \\
\hline 6 & 20.0 & 10 & 2.0 \\
\hline
\end{tabular}




\begin{tabular}{llll}
\hline 7 & 28.0 & 10 & 2.8 \\
\hline 8 & 32.0 & 10 & 3.2 \\
\hline 9 & 36.0 & 10 & 3.6 \\
\hline 10 & 40.0 & 10 & 4.0 \\
\hline 11 & 34.0 & 10 & 3.4 \\
\hline 12 & 28.0 & 10 & 2.8 \\
\hline 14 & 22.0 & 10 & 2.2 \\
\hline 15 & 16.0 & 10 & 1.6 \\
\hline 16 & 10.0 & 10 & 1.0 \\
\hline 35 & 4.0 & 10 & 0.4 \\
\hline
\end{tabular}

Figure 35 presents the failure type of this joint rock slope. It can be noticed that the blocks can be distinguished into three groups as based on the following behavior:

(1) Stability blocks; Blocks 14, 15 and 16 which are in the upper of the slope are stable, because the friction angle of the base of the blocks $\phi_{f}$ is larger than the angle of the base of block $\phi_{p}$ and these blocks are short enough to have their gravity centers lie inside the base. The formulas of the criteria for two stable conditions which prevent blocks from sliding and toppling respectively are given by:

$$
\begin{gathered}
\phi_{p}<\phi_{f} \\
\frac{y}{\Delta x}<\cot \phi_{p}
\end{gathered}
$$

(2) Toppling blocks; Blocks 4 to 13 compose the zone of toppling. On the basis of the limit equilibrium conditions the interaction between the blocks can be obtained. From block 5 to block 13, as the ratio $y_{n} / \Delta x$ is greater than $\cot \phi_{p}$, the toppling occurs. Block 4 still topples, although the ratio of the block satisfies Eq. (25). This is caused by the imbalance torque applied on this block.

(3) Sliding blocks; Blocks 1, 2 and 3 belong to the sliding group because the thrust force on the upper face of these blocks is greater than the friction force acting on the base of the block. The behavior modes of the blocks are shown in Table 5. The simulation result is shown in Figure 35. 


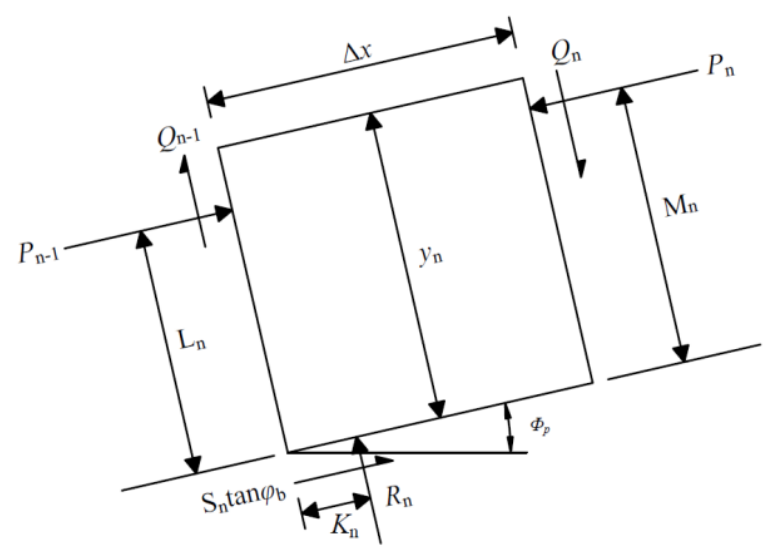

Figure 33. Limit equilibrium conditions for toppling/sliding of the $n^{\text {th }}$ block [63].

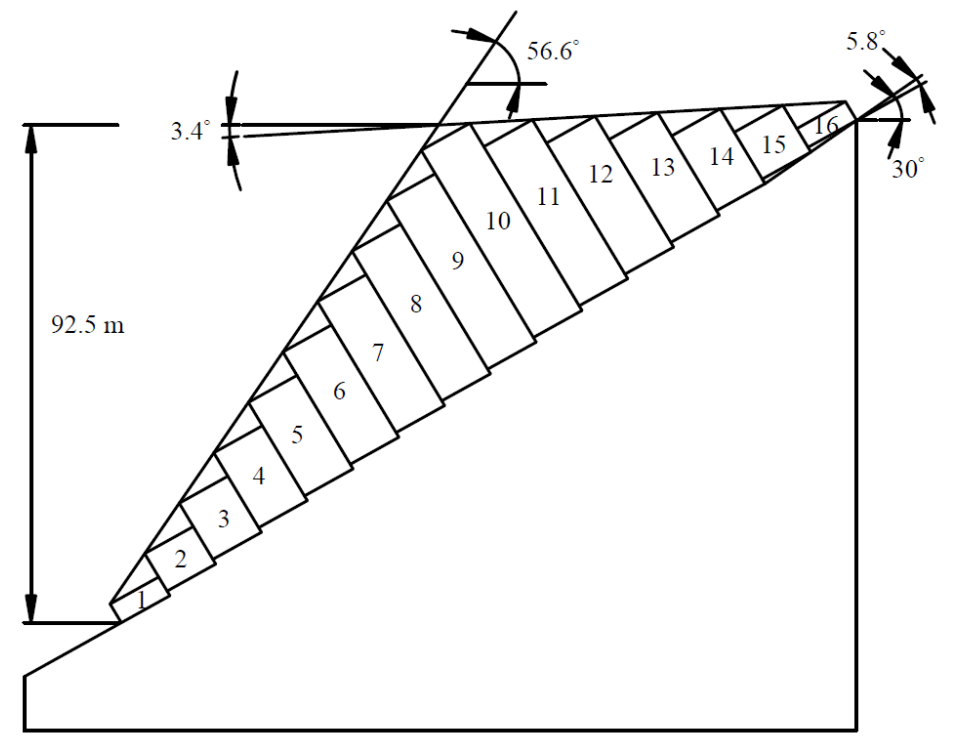

Figure 34. Model of sliding/toppling test of a joint rock slope.

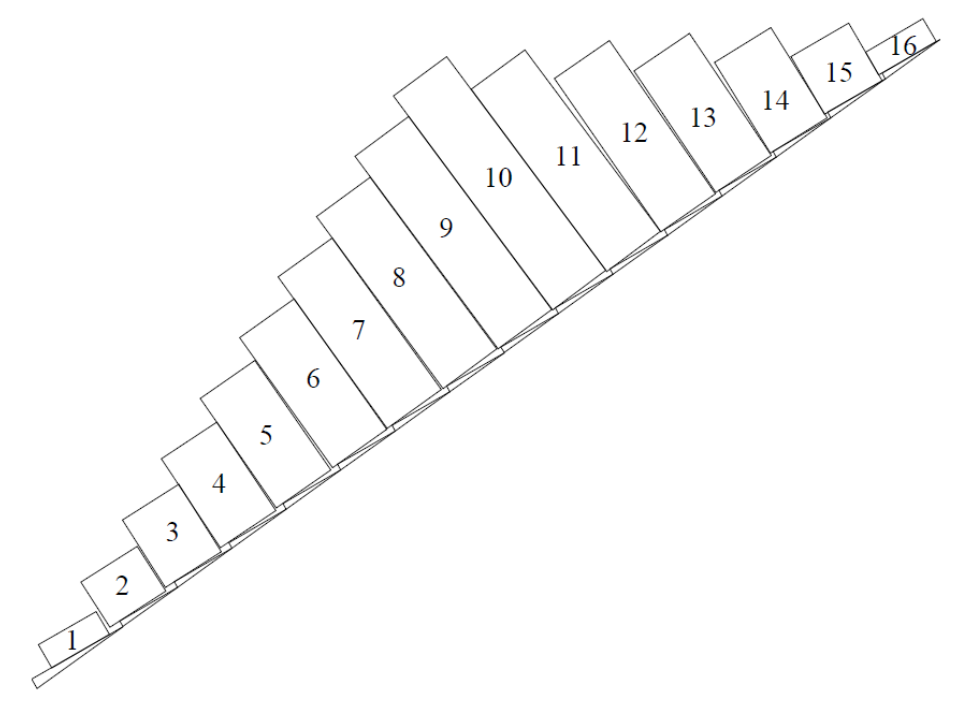


Figure 35. Simulation result of the sliding/toppling test of a joint rock slope by the proposed method.

Table 5

Simulated results by proposed method as related to Figure 35.

\begin{tabular}{cccccccc}
\hline$n$ & Mode & $n$ & Mode & $n$ & Mode & $n$ & Mode \\
\hline 1 & Sliding & 5 & Toppling & 9 & Toppling & 13 & Toppling \\
\hline 2 & Sliding & 6 & Toppling & 10 & Toppling & 14 & Stable \\
\hline 3 & Sliding & 7 & Toppling & 11 & Toppling & 15 & Stable \\
\hline 4 & Toppling & 8 & Toppling & 12 & Toppling & 16 & Stable \\
\hline
\end{tabular}

\subsection{Simulation of the joints structure effects on the sliding rock mass}

In this section, the accuracy verification of this proposed method is performed with the following simulation. This simulation is based on the experiment operated by Li [64] and the result is compared against the experiment data and available numerical results.

The simulation consists of five manual accumulation slopes with different sets of transfixion plane as shown in Table 6, and the geometrical properties of the slopes are listed in Table 7.

Table 6

Models of five manual accumulation slopes with different sets of transfixion plane:

\begin{tabular}{cc|c|c}
\hline Number & Diagram of the mechanism & Boints construction \\
\hline & & & \\
\hline
\end{tabular}


Table 7

The geometrical properties of the blocks

\begin{tabular}{ccc}
\hline Block type & Length $(\mathrm{m})$ & Height $(\mathrm{m})$ \\
\hline Big block & 0.2 & 0.1 \\
\hline Medium block & 0.1 & 0.1 \\
\hline Small block & 0.05 & 0.05 \\
\hline
\end{tabular}

In the experiment, five types of accumulation slope are placed on a platform, respectively. Then turning the platform counterclockwise with a constant angular velocity until the failure of slope occurs. The friction angle and cohesion of the blocks and the platform are the same which are set to be $\varphi_{\mu}=26$ and $c=2.14 \mathrm{~Pa}$. The constant angular velocity of platform is $0.03 \mathrm{rad} / \mathrm{s}$ and the vertical acceleration due to gravity is $9.81 \mathrm{~m} / \mathrm{s}^{2}$.

Table 8 shows the simulation results of five types of accumulation slopes and Figure 36 presents the failure behavior of the first accumulation slope. Table 9 compares the failure modes and failure angle between the current simulation and the previous results provided by $\mathrm{Li}$ [64]. It can be seen that the simulation result is in good agreement with the existing experimental and numerical data.

\section{Table 8}

The simulation results of five types of accumulation slopes.

Number of
simulation




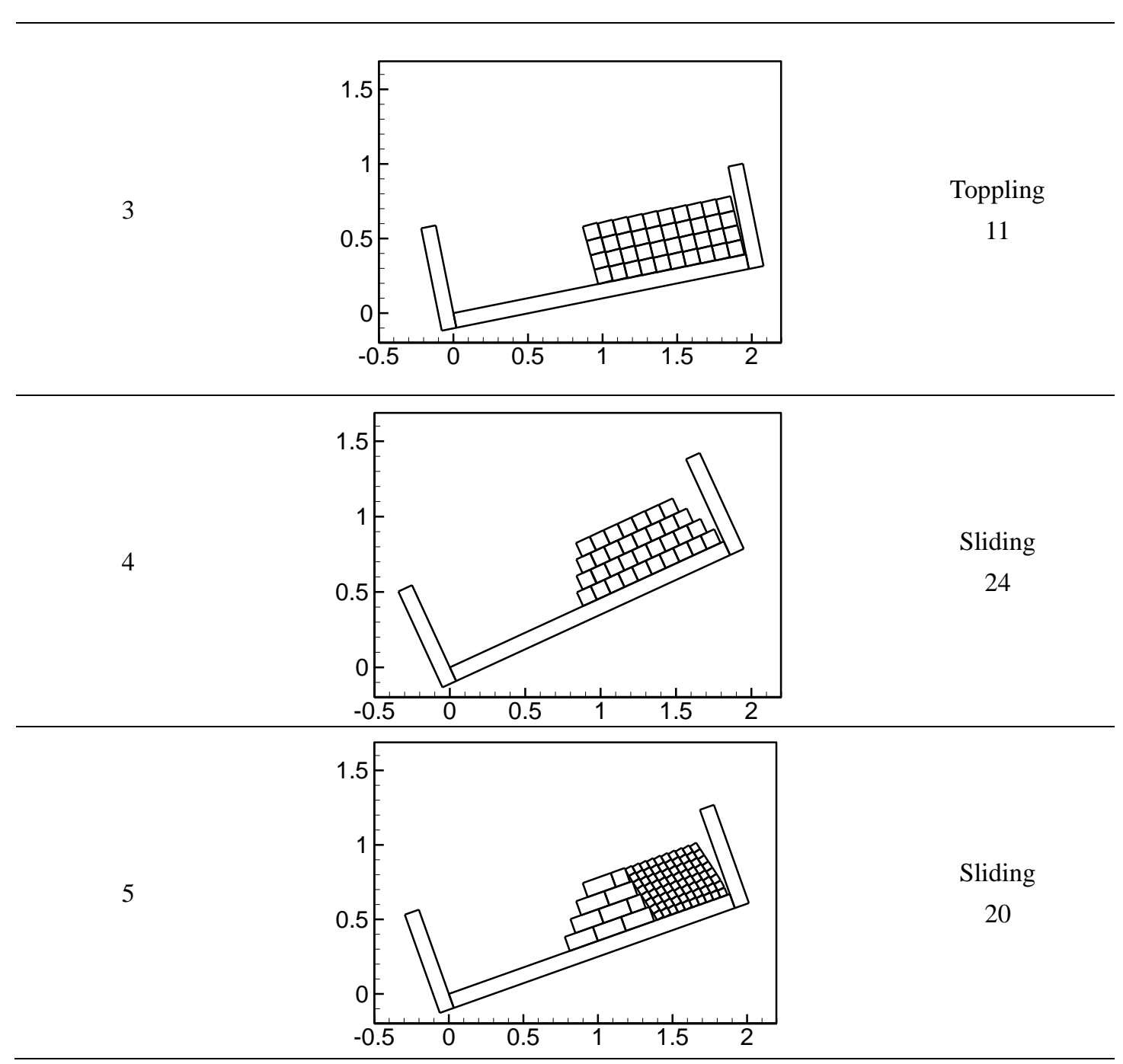

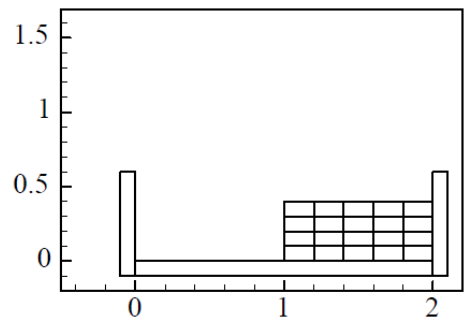

(a)

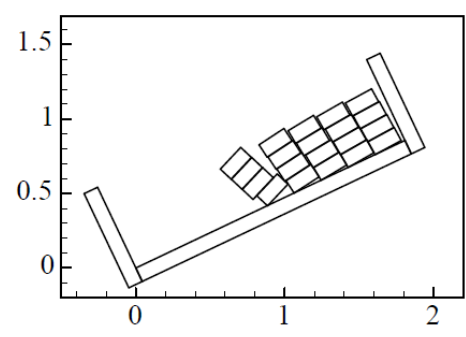

(d)

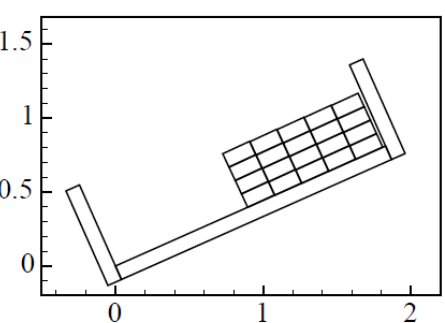

(b)

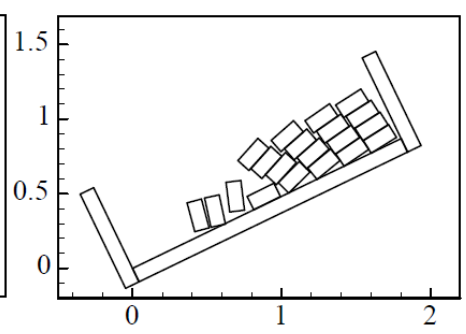

(e)

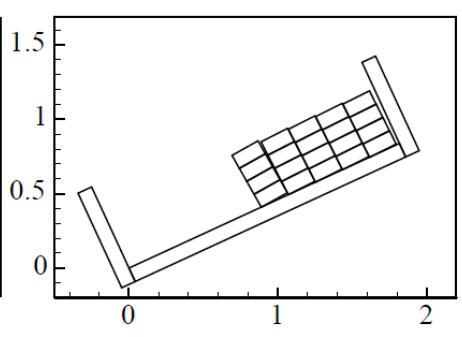

(c)

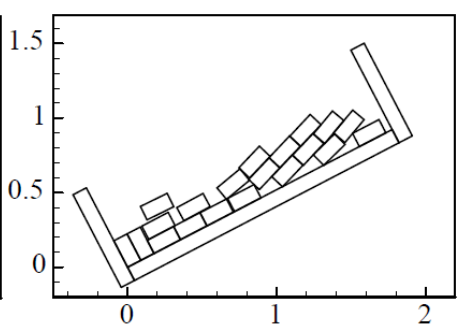

(f)

Figure 36. Failure process of the first slope which consists of big blocks with persistent joints; (a) $t=0$ 


$$
\text { s; (b) } t=13.93 \mathrm{~s} ; \text { (c) } t=14.4 \mathrm{~s} \text {; (d) } t=14.76 \mathrm{~s} \text {; (e) } t=14.99 \mathrm{~s} \text {; (f) } t=16 \mathrm{~s} \text {. }
$$

Table 9

Failure modes and failure angles of the five accumulation slopes.

\begin{tabular}{ccccccc}
\hline & Failure mode & 1 & 2 & 3 & 4 & 5 \\
\cline { 2 - 6 } & Toppling & Sliding & Toppling & Sliding & Sliding \\
\hline \multirow{2}{*}{$\begin{array}{c}\text { Distance potential function } \\
\text { method (deg) }\end{array}$} & 24.0 & 26.0 & 11.0 & 24.0 & 21.0 \\
\cline { 2 - 6 } \begin{tabular}{c} 
angle \\
\cline { 2 - 6 }
\end{tabular} & $\begin{array}{c}\text { Experiment data of Li } \\
(\mathrm{deg})\end{array}$ & $22.1 \sim 24.2$ & $25.0 \sim 26.3$ & $9.8 \sim 11.6$ & $23.0 \sim 24.9$ & $19.5 \sim 21.8$ \\
\cline { 2 - 6 } & $\begin{array}{c}\text { Numerical result of Li } \\
(\mathrm{deg})\end{array}$ & 23.0 & 25.5 & 10.0 & 23.5 & 20.0 \\
\hline
\end{tabular}

\subsection{Simulation of the Tangjiashan landslide}

Landslide is one of the common disasters in nature. In recent years, with the development of the discontinuous method, there is an extraordinary progress in the stability analysis for the rock slope in engineering. In 2008, the Wenchuan Earthquake, one of the major natural disasters in China, caused serious earthquake-induced disasters and among them the Tangjiashan landslide which has the largest potential risk due to its large volume. At present, some researches have focused on the analysis of the mechanism for the Tangjiashan landslide [65-67]. In this paper, a 2-D numerical model is adopted to analyze the kinetic movement process of the landslide.

In this simulation, the slope is set to be consisted of strong weathered rock masses, and the bedrock of the Tangjiashan slope is weakly weathered rock masses. The structure planes are divided into the bedding plane and joint plane (Figure 37). The physical-mechanical parameters of the rock masses used in this simulation are listed in Table 10 and the parameters of the structural planes are shown in Table 11.

Figure 38 illustrates the geometry of the model. According to the geologic data of the Tangjiashan landslide, the slope is discretized along the joint altitude as shown in Figure 39.

Figure 40 shows the sliding process of the landslide. It can be seen that the coverage of the landslide and the pattern of the barrier dam are in high degree of uniformity with the post-earthquake investigation by $\mathrm{Hu}$ [68] shown in Figure 37 and the simulation results by Cao [65]. 


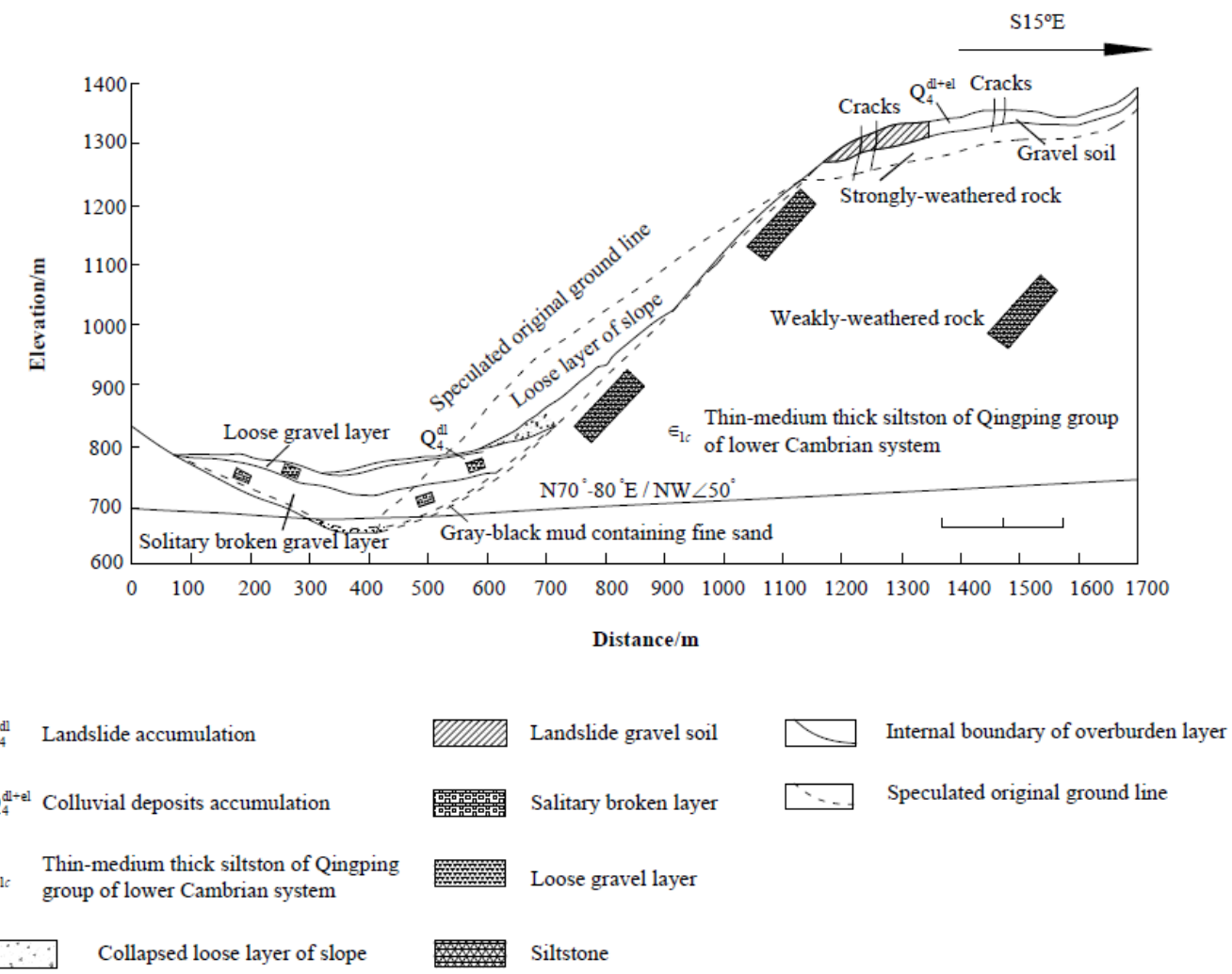

Figure 37. Engineering geology of Tangjiashan landslide and its barrier dam by Hu [68].

Table 10

The physical-mechanical parameters of the rock masses.

\begin{tabular}{cccccc}
\hline Rock mass & $\begin{array}{c}\text { Density } \\
\left(\mathrm{kg} / \mathrm{m}^{3}\right)\end{array}$ & $\begin{array}{c}\text { Cohesion } \\
(\mathrm{MPa})\end{array}$ & $\begin{array}{c}\text { Internal } \\
\text { friction } \\
\text { angle }(\mathrm{deg})\end{array}$ & $\begin{array}{c}\text { Bulk modulus } \\
(\mathrm{GPa})\end{array}$ & $\begin{array}{c}\text { Shear } \\
\text { modulus } \\
(\mathrm{GPa})\end{array}$ \\
\hline $\begin{array}{c}\text { Strongly } \\
\text { weathered } \\
\text { rock masses }\end{array}$ & 2550 & 1.28 & 32 & 1.3 & 0.8 \\
\hline $\begin{array}{c}\text { Weakly } \\
\text { weathered } \\
\text { rock masses }\end{array}$ & 2650 & 2.34 & 35 & 1.2 & 0.8 \\
\hline
\end{tabular}

Table 11

The mechanical parameters of the structural planes.

\begin{tabular}{cccccc}
\hline $\begin{array}{c}\text { Structural } \\
\text { plane }\end{array}$ & $\begin{array}{c}\text { Normal } \\
\text { stiffness } \\
(\mathrm{GPa} / \mathrm{m})\end{array}$ & $\begin{array}{c}\text { Shear } \\
\text { stiffness } \\
(\mathrm{GPa} / \mathrm{m})\end{array}$ & $\begin{array}{c}\text { Internal } \\
\text { friction angle } \\
(\mathrm{deg})\end{array}$ & $\begin{array}{c}\text { Cohesion } \\
(\mathrm{MPa})\end{array}$ & $\begin{array}{c}\text { Tensile } \\
\text { strength } \\
(\mathrm{MPa})\end{array}$ \\
\hline Bedding plane & 3.6 & 1.2 & 30 & 0.5 & 0.05 \\
\hline Joint plane & 3.6 & 1.2 & 23 & 0.3 & 0.03 \\
\hline
\end{tabular}




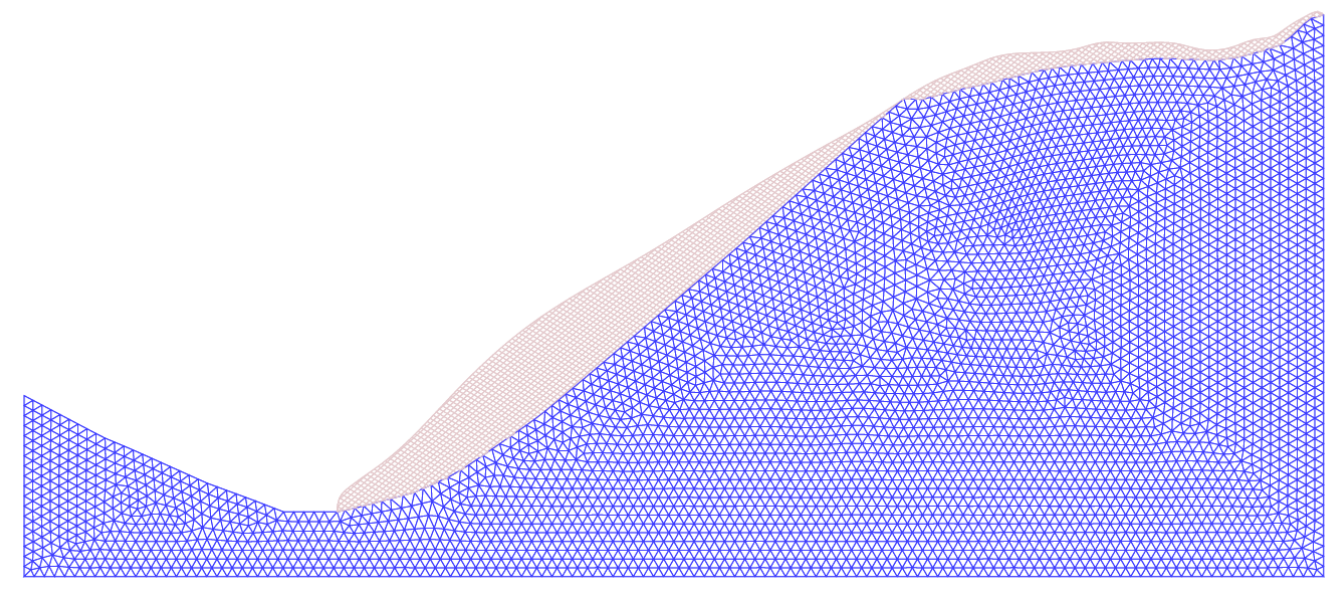

Figure 38. Numerical simulation model of Tangiiashan slope.

Figure 39. Discrete element types of the slope. 


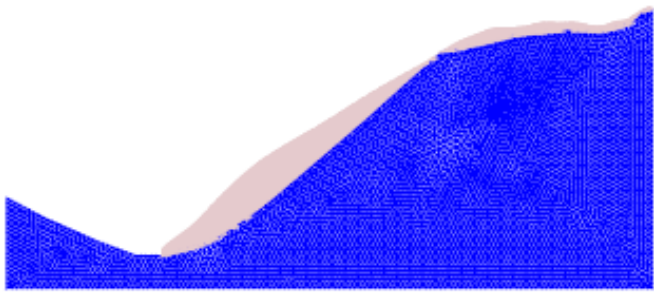

(a)

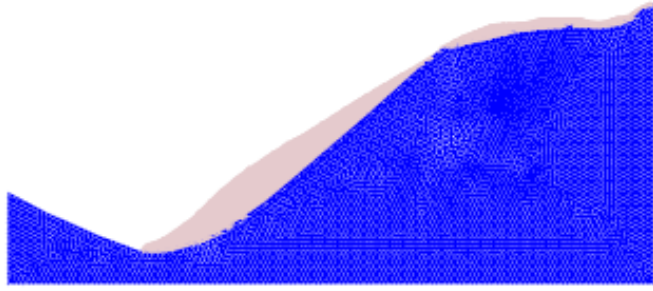

(c)

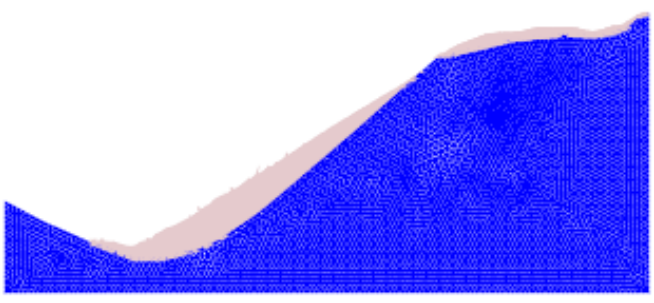

(e)

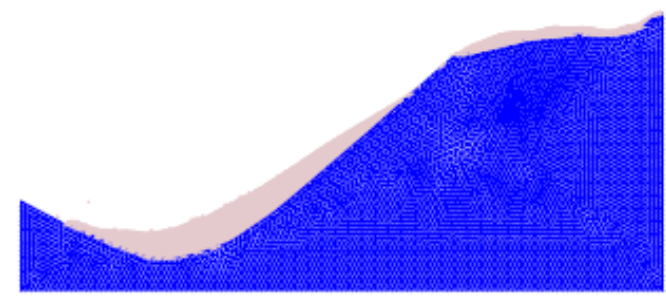

(g)

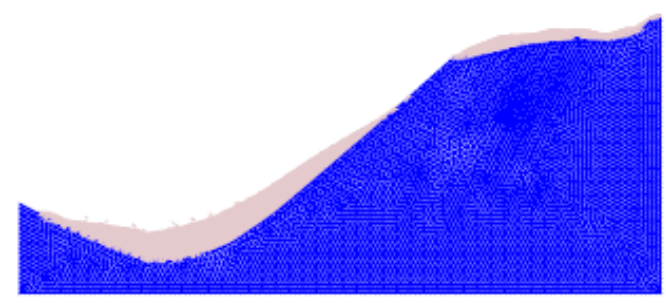

(i)

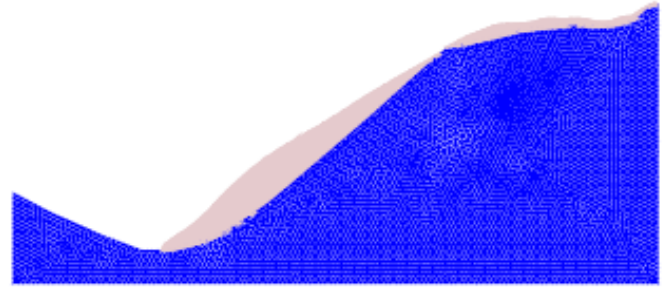

(b)

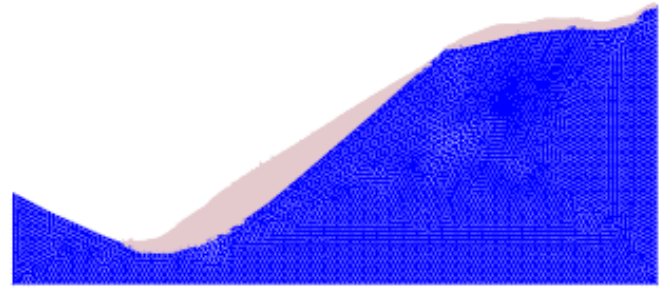

(d)

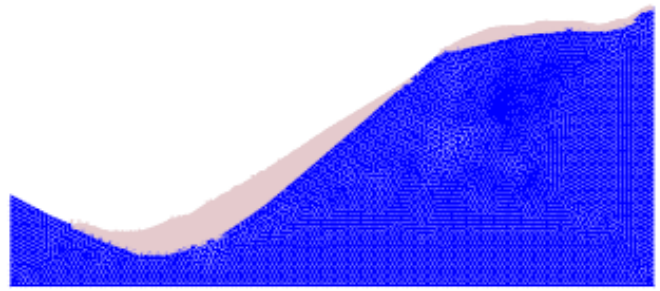

(f)

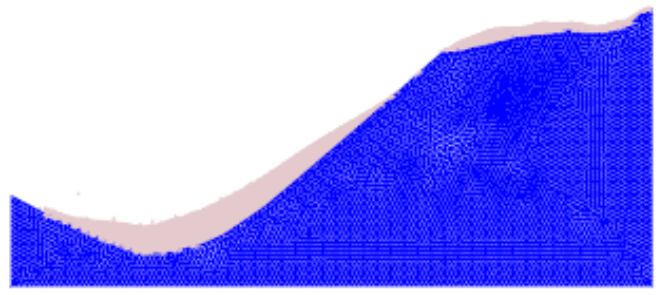

(h)

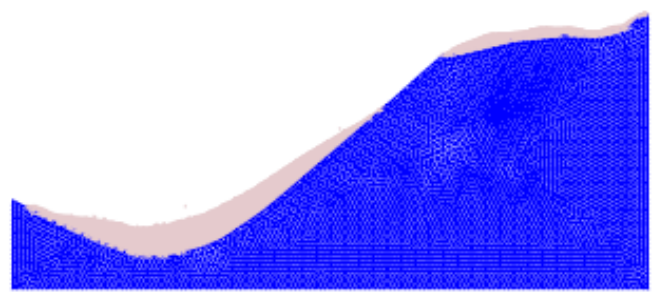

(1)

Figure 40. Evolution of the landslide using the newly proposed method of the distance potential function at different time stages (a) $t=0 \mathrm{~s}$; (b) $t=0.5 \mathrm{~s}$; (c) $t=1.0 \mathrm{~s}$; (d) $t=1.5 \mathrm{~s}$; (e) $t=2.0 \mathrm{~s}$; (f) $t=$ $2.5 \mathrm{~s} ;(\mathrm{g}) t=3.0 \mathrm{~s} ;$ (h) $t=3.5 \mathrm{~s}$; (i) $t=4.0 \mathrm{~s}$; and (j) $t=5.0 \mathrm{~s}$.

\section{Conclusions}

Our definition of distance potential function has been seen to be appropriate for arbitrary twodimensional convex elements used in discrete element model. A complete normal contact force 
calculation model, which includes the magnitude, direction, and the normal contact force moment, was determined under the concept of the new potential definition. It led to a new fundamental algorithm for the tangential contact force. A new non-uniform block contact detection algorithm was also introduced for arbitrary convex polygonal discrete elements with different sizes. This computational method is clear, efficient, and stable for 2D models as illustrated for several benchmark problems.

To be specific, the main features of the proposed method can be summarized as follows:

(1) Instead of using the standard potential function, the normal contact force is calculated using the definition of a distance potential function. It gives an accurate potential value and normal contact force without being adversely affected by the element shape.

(2) Compared with the FDEM, the proposed method can be implemented to deal with the problems involving a large number of arbitrary polygonal elements.

(3) The proposed algorithm for the tangential contact force makes the results more accurate and reliable.

(4) A non-uniform block discrete element contact detection algorithm is introduced to solve the contact detection problem for arbitrary convex elements with different sizes.

The effectiveness of the method for the numerical simulation using the distance potential for a system of arbitrary convex polyhedral elements will be discussed in the future study.

\section{Acknowledgement}

This work was supported by the National Natural Science Foundation of China (Grant No. 51279050), the 15th Fok Ying-Tong Education Foundation for Young Teachers in the Higher Education Institutions of China (Grant No. 151073), the National Key R \& D Program of China (Grant No. 2016YFC0401601), the project funded by the Priority Academic Program Development of Jiangsu Higher Education Institutions (YS11001), and the 111 Project.

\section{References}

1. Shi GH, Goodman RE. Two dimensional discontinuous deformation analysis. International Journal for Numerical \& Analytical Methods in Geomechanics 1985; 9:541-556.

2. Koo CY, Chern JC. Modification of the DDA method for rigid block problems. International Journal of Rock Mechanics \& Mining Sciences 1998; 35:683-693.

3. Zhao Z, Gu J. Stress recovery procedure for discontinuous deformation analysis. Advances in Engineering Software 2007; 40:52-57.

4. Bao H, Zhao Z. The vertex-to-vertex contact analysis in the two-dimensional discontinuous deformation analysis. Advances in Engineering Software 2012; 45:1-10.

5. Ning Y, Yang J, An X, Ma G. Modelling rock fracturing and blast-induced rock mass failure via advanced discretisation within the discontinuous deformation analysis framework. Computers \& Geotechnics 2011 ; 38:40-49. 
6. Zhang Y, Chen G, Zheng L, Li Y, Wu J. Effects of near-fault seismic loadings on run-out of large-scale landslide: A case study. Engineering Geology 2013; 166:216-236.

7. Shi GH. Application of discontinuous deformation analysis on stability analysis of slopes and underground power houses. Geomechanics \& Geoengineering 2014; 9:80-96.

8. Morgan WE, Aral MM. An implicitly coupled hydro-geomechanical model for hydraulic fracture simulation with the discontinuous deformation analysis. International Journal of Rock Mechanics \& Mining Sciences 2015; 73:82-94.

9. Zheng H, Zhang P, Du X. Dual form of discontinuous deformation analysis. Computer Methods in Applied Mechanics \& Engineering 2016; 305:196-216.

10. $\mathrm{Li} \mathrm{XK}$, Zheng $\mathrm{H}$. Condensed form of complementarity formulation for discontinuous deformation analysis. Science China Technological Sciences 2015; 58:1509-1519.

11. Jing L. A review of techniques, advances and outstanding issues in numerical modelling for rock mechanics and rock engineering. International Journal of Rock Mechanics \& Mining Sciences 2003; 40:283-353.

12. Shi GH. Manifold method of material analysis. Trans.army Conf.on Appl.math. \& Comp.u.s.army Res.office. 1991; 57-76.

13. Terada K, Asai M, Yamagishi M. Finite cover method for linear and non-linear analyses of heterogeneous solids. International Journal for Numerical Methods in Engineering 2003; 58:1321-1346.

14. Terada K, Ishii T, Kyoya T, Kishino Y. Finite cover method for progressive failure with cohesive zone fracture in heterogeneous solids and structures. International Journal for Numerical Methods in Engineering 2007; 58:1321-1346.

15. Ma GW, An XM, Zhang HH, Li LX. Modeling complex crack problems using the numerical manifold method. International Journal of Fracture 2009; 156:21-35.

16. Zheng H, Xu DD. New strategies for some issues of numerical manifold method in simulation of crack propagation. International Journal for Numerical Methods in Engineering 2014; 97:986-1010.

17. Ma GW, An XM, He L. The numerical manifold method: a review. International Journal of Computational Methods 2010; 7:1-32.

18. Smeets B, Odenthal T, Vanmaercke S, Ramon H. Polygon-based contact description for modeling arbitrary polyhedra in the discrete element method. Computer Methods in Applied Mechanics \& Engineering 2015; 290:277-289.

19. Höhner D, Wirtz S, Kruggel-Emden H, Scherer V. Comparison of the multi-sphere and polyhedral approach to simulate non-spherical particles within the discrete element method: Influence on temporal force evolution for multiple contacts. Powder Technology 2011; 208:643656.

20. Cundall PA, Strack ODL. A discrete numerical model for granular assemblies. Géotechnique 1979; 29:47-65.

21. Egholm DL, Sandiford M, Clausen OR, Nielsen SB. A new strategy for discrete element numerical models: 2. sandbox applications. Journal of Geophysical Research: Solid Earth 2007; 112:1-12.

22. Jung JW, Santamarina JC, Soga K. Stress-strain response of hydrate-bearing sands: numerical study using discrete element method simulations. Journal of Geophysical Research: Solid Earth 2012; 117:531-535. 
23. McDowell GR. Discrete element modelling of soil particle fracture. Géotechnique 2015; 52:131-136.

24. Al-Busaidi A, Hazzard JF, Young RP. Distinct element modeling of hydraulically fractured lac du bonnet granite. Journal of Geophysical Research: Solid Earth 2005; 110:351-352.

25. Ghazvinian A, Sarfarazi V, Schubert W, Blumel M. A study of the failure mechanism of planar non-persistent open joints using PFC2D. Rock Mechanics \& Rock Engineering 2012; 45:677693.

26. Mollon G, Richefeu V, Villard P, Daudon D. Numerical simulation of rock avalanches influence of a local dissipative contact model on the collective behavior of granular flows. Journal of Geophysical Research: Earth Surface 2012; 117:191-204.

27. Latham JP, Munjiza A. The modelling of particle systems with real shapes. Philosophical Transactions of The Royal Society of London 2004; 362:1953-1972.

28. Oda M, Konishi J, Nemat-Nasser S. Experimental micromechanical evaluation of strength of granular materials: effects of particle rolling. Mechanics of Materials 1982; 1:269-283.

29. Lin XS, Ng TT. Contact detection algorithms for three-dimensional ellipsoids in discrete element modelling. International Journal for Numerical \& Analytical Methods in Geomechanics 1995; 19:653-659.

30. Jiang MJ, Shen ZF, Wang JF. A novel three-dimensional contact model for granulates incorporating rolling and twisting resistances. Computers \& Geotechnics 2015; 65:147-163.

31. Cundall PA. A computer model for simulating progressive, large-scale movements in blocky rock systems. Proceedings of the Symposium of the International Society of Rock Mechanics 1971; 1:8-11.

32. Cundall PA. Formulation of a three-dimensional distinct element model - part I: a scheme to detect and represent contacts in a system composed of many polyhedral blocks. International Journal of Rock Mechanics \& Mining Sciences \& Geomechanics Abstracts 1988; 25:107-116.

33. Cundall PA. UDEC - a generalised distinct element program for modelling jointed rock. U.S. Army European Research Office and Defence Nuclear Agency, Contract Report 1980; DAJA 3739-C-0548.

34. Cappa F, Guglielmi Y, Soukatchoff VM, Mudry J, Bertrand C,Charmoille A. Hydromechanical modeling of a large moving rock slope inferred from slope levelling coupled to spring longterm hydrochemical monitoring: example of the La Clapière landslide (Southern Alps, France). Journal of Hydrology 2004; 291:67-90.

35. Zhu JB, Deng XF, Zhao XB. A numerical study on wave transmission across multiple intersecting joint sets in rock masses with UDEC. Rock Mechanics \& Rock Engineering 2013; 46:1429-1442.

36. Zhao XB, Zhao J, Cai JG,Hefny AM. UDEC modelling on wave propagation across fractured rock masses. Computers and Geotechnics 2008; 35:97-104.

37. Houlsby GT, Boon CW, Utili S. New insights into the 1963 Vajont slide using 2D and 3D distinct-element method analyses. Géotechnique 2014; 64:800-816.

38. Munjiza A. The combined finite-discrete element method. England: Wiley \& Sons 2004.

39. Feng YT, Owen DRJ. A 2D polygon/polygon contact model: algorithmic aspects. Engineering Computations 2004; 21:265-277.

40. Feng YT, Han K, Owen DRJ. Energy-conserving contact interaction models for arbitrarily shaped discrete elements. Computer Methods in Applied Mechanics \& Engineering 2012; $205-$ 
208:169-177.

41. Nassauer B, Liedke T, Kuna M. Polyhedral particles for the discrete element method. Granular Matter 2013; 15:85-93.

42. Nassauer B, Kuna M. Contact forces of polyhedral particles in discrete element method. Granular Matter 2013; 15:349-355.

43. Smeets B, Odenthal T. Modeling contact interactions between triangulated rounded bodies for the discrete element method. Computer Methods in Applied Mechanics \& Engineering 2014; 277:219-238.

44. Dong K, Wang C, Yu A. A novel method based on orientation discretization for discrete element modelling of non-spherical particles. Chemical Engineering Science 2015; 126:500-516.

45. Nezami EG, Hashash YMA, Zhao DW,Ghaboussi J. A fast contact detection algorithm for 3-D discrete element method. Computers \& Geotechnics 2004; 31:575-587.

46. Nezami EG, Hashash YMA, Zhao DW, Ghaboussi J. Shortest link method for contact detection in discrete element method. International Journal for Numerical \& Analytical Methods in Geomechanics 2006; 30:783-801.

47. Boon CW, Houlsby GT, Utili S. A new algorithm for contact detection between convex polygonal and polyhedral particles in the discrete element method. Computers \& Geotechnics 2012; 44:73-82.

48. Boon CW, Houlsby GT, Utili S. A new contact detection algorithm for three-dimensional nonspherical particles. Powder Technology 2013; 248:94-102.

49. $\mathrm{Wu} \mathrm{JH}$. New edge-to-edge contact calculating algorithm in three-dimensional discrete numerical analysis. Advances in Engineering Software 2008; 39:15-24.

50. Kodam M, Bharadwaj R, Curtis J, Hancock B, Wassgren C. Cylindrical object contact detection for use in discrete element method simulations. Part I - contact detection algorithms. Chemical Engineering Science 2010; 65:5852-5862.

51. Jin F, Xin HL, Zhang CH, Sun QC. Probability-based contact algorithm for non-spherical particles in DEM. Powder Technology 2011; 212:134-144.

52. Munjiza A, Owen DRJ, Bicanic N. A combined finite-discrete element method in transient dynamics of fracturing solids. International Journal of Engineering Computations 1995; 12:145-174.

53. Munjiza A, Andrews KRF. NBS contact detection algorithm for bodies of similar size. International Journal for Numerical Methods in Engineering 1998; 43:131-149.

54. Mahabadi OK, Grasselli G, Munjiza A. Y-GUI: a graphical user interface and pre-processor for the combined finite-discrete element code, Y2D, incorporating material heterogeneity. Computers \& Geosciences 2010; 36:241-252.

55. Munjiza A, John NWM. Mesh size sensitivity of the combined FEM/DEM fracture and fragmentation algorithms. Engineering Fracture Mechanics 2002; 69:281-295.

56. Wriggers P, Knackenhorst U. Analysis and simulation of contact problems. Springer Berlin Heidelberg 2006.

57. Munjiza A, Bangash A, John NWM. The combined finite-discrete element method for structural failure and collapse. Engineering Fracture Mechanics 2004; 71:469-483.

58. Mahabadi OK, Lisjak A, Munjiza A, Grasselli G. Y-Geo: new combined finite-discrete element numerical code for geomechanical applications. International Journal of Geomechanics 2012; 12:676-688. 
59. Rougier E, Bradley CR, Broom ST, Knight EE, Munjiza A, Sussman AJ,Swift RP. The combined finite-discrete element method applied to the study of rock fracturing behavior in 3D. 45th US Rock Mech. / Geomech. Symp. held San Fr. CA 2011; 26-29.

60. Rougier E, Knight EE, Broome ST, Sussman AJ, Munjiza A. Validation of a three-dimensional finite-discrete element method using experimental results of the split hopkinson pressure bar test. International Journal of Rock Mechanics \& Mining Sciences 2014; 70:101-108.

61. Munjiza A, Rougier E, John NWM. MR linear contact detection algorithm. International Journal for Numerical Methods in Engineering 2006; 66:46-71.

62. Goodman RE. Methods of geological engineering in discontinuous rocks. Mécanique Des Roches 1976.

63. Hoek E, Bray J. Rock slope engineering. Institution of Mining and Metallurgy 1977; 14:492494.

64. Li SH, Wang JG, Liu BS, Dong DP. Analysis of critical excavation depth for a jointed rock slope using a face-to-face discrete element method. Rock Mechanics \& Rock Engineering 2007; 40:331-348.

65. Cao YB, Dai F, Xu C, Tu X, Min H, Cui F. Discrete element simulation of deformation and movement mechanism for tangjiashan landslide. Chinese Journal of Rock Mechanics \& Engineering 2011; 30:2878-2887.

66. Cui F, Hu R, Yin R, Xu Q, Zhang M. Discrete element analysis of collapsing and sliding response of slope triggered by time difference coupling effects of $\mathrm{p}$ and s seismic waves - taking tangjiashan landslide in beichuan county for example. Chinese Journal of Rock Mechanics \& Engineering 2010; 29:319-327.

67. Luo G, Hu X, Gu C, Wang Y. Numerical simulations of kinetic formation mechanism of tangjiashan landslide. Journal of Rock Mechanics \& Geotechnical Engineering 2012; 2012:149-159.

68. HU XW, Huang R, Shi Y, LU XP, Zhu H,Wang X. Analysis of blocking river mechanism of tangjiashan landslide and dam-breaking mode of its barrier dam. . Chinese Journal of Rock Mechanics \& Engineering 2009; 28:181-189. 